\title{
Nuclear magnetic resonance relaxation due to the translational diffusion of fluid confined to quasi-two-dimensional pores
}

\author{
D. A. Faux, P. J. McDonald, and N. C. Howlett \\ Department of Physics, University of Surrey, Guildford, GU2 7XH, UK
}

\begin{abstract}
Nuclear magnetic resonance (NMR) relaxation experimentation is an effective technique for non-destructively probing the dynamics of proton-bearing fluids in porous media. The frequencydependent relaxation rate $T_{1}^{-1}$ can yield a wealth of information on the fluid dynamics within the pore provided data can be fit to a suitable spin diffusion model. A spin diffusion model yields the dipolar correlation function $G(t)$ describing the relative translational motion of pairs of ${ }^{1} \mathrm{H}$ spins which then can be Fourier transformed to yield $T_{1}^{-1} . G(t)$ for spins confined to a quasi-two-dimensional (Q2D) pore of thickness $h$ is determined using theoretical and Monte Carlo techniques. $G(t)$ shows a transition from three- to two-dimensional (2D) motion with the transition time proportional to $h^{2} . T_{1}^{-1}$ is found to be independent of frequency over the range 0.01-100 MHz provided $h \gtrsim 5 \mathrm{~nm}$ and increases with decreasing frequency and decreasing $h$ for pores of thickness $h<3 \mathrm{~nm}$. $T_{1}^{-1}$ increases linearly with the bulk water diffusion correlation time $\tau_{b}$ allowing a simple and direct estimate of the bulk water diffusion coefficient from the high-frequency limit of $T_{1}^{-1}$ dispersion measurements in systems where the influence of paramagnetic impurities is negligible. Monte Carlo simulations of hydrated Q2D pores are executed for a range of surfaceto-bulk desorption rates for a thin pore. $G(t)$ is found to decorrelate when spins move from the surface to the bulk, display three-dimensional properties at intermediate times and finally show a bulk-mediated surface diffusion (Lévy) mechanism at longer times. The results may be used to interpret NMR relaxation rates in hydrated porous systems in which the paramagnetic impurity density is negligible.
\end{abstract}

PACS numbers: 


\section{INTRODUCTION}

This is the first of two articles on the interpretation of proton nuclear magnetic resonance (NMR) relaxation experiments on fluids confined to the pore spaces of solid porous media. Systems and applications of interest include water and oil in rocks for oil recovery, zeolites for water desalination, purification and catalysis, clays with application to the containment of radioactive wastes, porous glasses, cement-based materials where water impacts strength and durability, polymers for fuel cells and wood for sustainable development [1-18]. Porous media are notoriously complex but results of NMR relaxometry experiments can be used in tandem with appropriate theoretical models to infer the dynamics and microstructure of the fluid and confining matrix at the nano-scale. Other techniques which can be useful for exploring morphology and proton diffusion include small-angle x-ray scattering [19-22] and quasi-elastic or small-angle neutron scattering [23-27].

Nuclear magnetic resonance relaxometry measures the nuclear spin-lattice (longitudinal) or spin-spin (transverse) relaxation times of ${ }^{1} \mathrm{H}$ nuclei, $T_{1}$ or $T_{2}$ respectively. The NMR relaxation times are governed by modulations of the local magnetic field experienced by the resonant nuclei caused by the relative motion of pairs of spins. The relaxation times of fluid molecules adsorbed to the pore surface or encountering a surface through diffusion are much shorter than those of the corresponding bulk fluid. Specific NMR techniques applied to porous media include relaxation rate dispersion measurements obtained at low NMR frequencies [3-14], pulsed-field-gradient measurements which yield macroscopic fluid flow and diffusion properties (see an excellent review by Gladden and Mitchell [16]), $T_{1}-T_{2}$ spin correlation measurements and $T_{2}-T_{2}$ spin exchange measurements which provide information on pore filling and emptying times $[10,15,16,18]$. A review of the scope of NMR relaxometry as applied to porous systems, including results on carbonate rock, cementitious material, porous glass and other systems, is presented by Korb [10].

The NMR dispersion (NMRD) technique is a particularly valuable probe of molecular dynamics. NMRD measures $T_{1}^{-1}$ as a function of NMR frequency in the low-frequency range $(\mathrm{kHz}$ to $\mathrm{MHz})$ allowing access to dynamical processes occurring over ps- $\mu$ s time scales therefore providing information on the nano-scale dynamics of fluid contained in pores. Highquality NMRD measurements of a range of porous systems have been reported [3-7, 10-13]. 
Deducing the dynamical properties of a confined fluid from a NMRD measurement is not straightforward however. It is necessary to devise a model for the frequency dependence of $T_{1}^{-1}$ which can be fit to the experimental dispersion. To achieve this, the spin dynamics must be described by an appropriate diffusion model from which the dipolar correlation function, $G(t)$ is calculated. The Fourier transform of $G(t)$ straightforwardly yields the $T_{1}^{-1}$ dispersion which can be fit to the experimental NMRD data. $G(t)$ is a measure of the time-dependence of the dipolar interaction of pairs of spins which are moving relative to each other and the theoretical challenge lies in the determination of $G(t)$ for the model of choice.

The first successful theoretical description of the relaxation rate dispersion was provided by Bloembergen, Purcell and Pound [28], commonly referred to as BPP theory, for a bulk liquid (glycerin) in which $G(t)$ decays exponentially. The complexity of the theoretical treatments increases significantly for fluids subject to the influence of surfaces. Levitz and co-workers have explored surface-mediated diffusion models chiefly for biological systems [29-31] and Kimmich and co-workers [1, 2, 5, 12], prompted by the work of Bychuk and O'Shaughnessy [32, 33], developed a model of a fluid in which molecules desorb from a surface, diffuse in a bulk-like layer close to the surface, before returning to the surface. This leads to Lévy dynamics and is known as bulk-mediated surface diffusion (BMSD). The most generally-applicable theoretical descriptions for the relaxation rate dispersion for fluids in porous media, however, has been developed by Korb and co-workers [3, 6-11, 15, 34], which we refer to generically as the Korb models. The Korb models are usually applied to systems in which the dominant relaxation mechanism is due to the heteronuclear interaction of electronic spins due to paramagnetic ion impurities (such as $\mathrm{Fe}^{3+}$ or $\mathrm{Mn}^{2+}$ ) in the confining solid and fluid protons in the pores.

The Kimmich, Levitz and Korb families of models are examples of models which simplify the complex fluid dynamics but which capture the essential physical processes to successfully describe NMRD data. These are variants of a general model illustrated in Fig. 1 for a fluidfilled quasi-two-dimensional (Q2D) pore system. Three classes of spins are identified as follows: fixed electronic paramagnetic impurity spins located in the crystal (labelled $\sigma$ ), slow-moving liquid proton spins contained in a monolayer at the surface (labelled $\ell$ ) and bulk liquid proton spins (labelled $b$ ). The complex spin dynamics of the mobile spins are characterised by the various models by one or more of three time constants $\tau_{\ell}, \tau_{d}$ and $\tau_{b}$. $\tau_{\ell}$ 
and $\tau_{b}$ are the characteristic diffusion correlation times for spins in the surface layer or bulk respectively. These diffusion correlation times are related to the diffusion coefficient $D$ by

$$
D=\frac{\delta^{2}}{6 \tau}
$$

so that $\tau$ (which is either $\tau_{\ell}$ or $\tau_{b}$ ) is the mean time for a spin to move a distance $\delta$ in $3 \mathrm{D}$, where $\delta$ is a convenient molecular-scale distance. For consistency with previous work, we set $\delta$ as the approximate distance between water molecules, that is $\delta=0.27 \mathrm{~nm}$, and this value is used regardless of the nature of the actual mobile fluid. For example, $\tau_{b}=5.3$ ps for pure water at room temperature [35] and $\tau_{b} \approx 10$ ps for low-molecular-mass liquid alkanes [36]. $\tau_{d}$ is the desorption time which is the time after which a fraction $e^{-1}$ of spins remains in the surface layer.

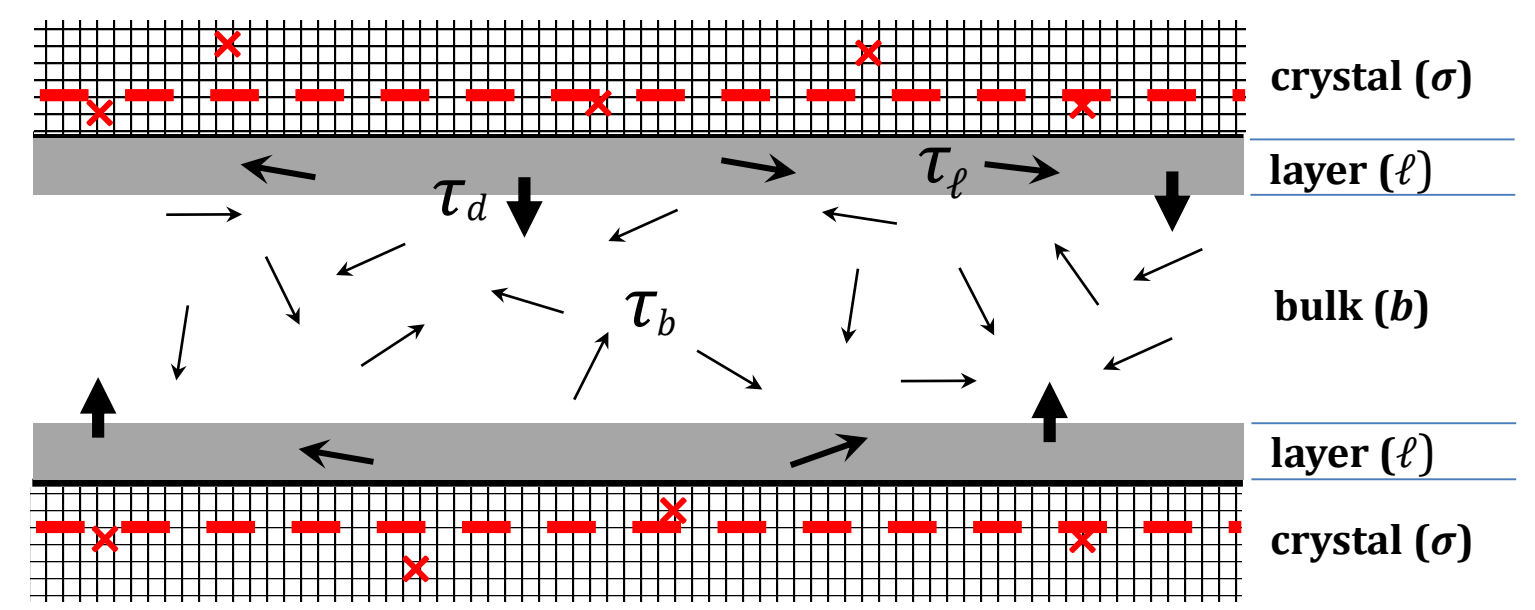

FIG. 1: The model structure of a Q2D pore is presented showing rare paramagnetic impurities in the crystal $(X)$ with the dashed line representing an equivalent single layer of paramagnetic impurities of uniform density. A layer of slow-moving fluid $(\ell)$ and bulk $(b)$ fluid are shown with diffusion correlation times $\tau_{\ell}$ and $\tau_{b}$ respectively. The desorption of spins from the surface layer to the bulk is characterised by the time $\tau_{d}$.

Various models can be incorporated into the framework of the general model illustrated in Fig. 1, which we refer to as the $3 \tau$ model, using just those elements that capture interactions sufficient to describe the relaxation rate dispersion for a particular system. This article addresses the relaxation associated with the relative motion of proton spins in the bulk of the pore. The consideration of the interaction of mobile spins with paramagnetic impurities 
is deferred to the following paper [37], referred to hereafter as Paper II. Fast-field-cycling dispersion measurements of porous systems provide a wealth of information on the dynamics of confined fluid in systems where the paramagnetic impurity concentration is negligible. Systems include certain porous glasses, plasters, carbonates and synthetic clays $[5,10,12]$.

We present the first calculation of the bulk-bulk relaxation rate, which we label $T_{1, b b}^{-1}$, for spins diffusing in a Q2D pore. In Paper II, these results contribute to the re-analysis of previously-published dispersion data for a plaster paste and a synthetic saponite clay. The choice of Q2D pore illustrated in Fig. 1, in which the fluid is confined in one direction and unbounded in the plane, is found to provide a quantitative estimate of the bulk diffusion correlation time $\tau_{b}$ when fit to experimental dispersion curves. Both plaster and saponite present flat surfaces to the surface fluid.

The calculation of $T_{1, b b}^{-1}$ for a Q2D pore requires, first, the determination of the probability density function describing the relative motion of pairs of mobile spins in a confined space. Details of this calculation are provided in Appendix A and validated by Monte Carlo (MC) simulation in Sec. III. The result for the spin-pair probability density function is then used to determine $T_{1, b b}^{-1}$ with details presented in Appendix B. The evaluation of $T_{1, b b}^{-1}$ allows, for the first time, a quantitative estimate of the bulk fluid diffusion correlation time $\tau_{b}$ from $T_{1}^{-1}$ dispersion measurements. In the second part of this paper, presented in Sec. III, a MC method is used to calculate the dipolar correlation function $G(t)$ for a Q2D pore which incorporates both surface and bulk environments for pore water including the complex dynamics of layer-bulk exchange. The MC simulations allow a numerical estimate of $T_{1, b \ell}^{-1}$ and $T_{1, \ell \ell}^{-1}$ for Q2D pores. All results are collectively presented in Sec. IV with conclusions drawn in Sec. V.

\section{THEORY}

\section{A. The relaxation rates $T_{1}^{-1}$ and $T_{2}^{-1}$}

Expressions are derived which allow the calculation of the spin-lattice and spin-spin relaxation rates, $T_{1}^{-1}$ and $T_{2}^{-1}$ respectively, for fluid proton spins confined to a Q2D pore space 
of uniform spin density. The bulk fluid is unbounded in the plane lying parallel to the hardwall boundaries and is confined to a region of thickness $h$. Pore surfaces are assumed to act as reflecting boundaries. In other words, whilst the crystal surfaces contain a surface layer of fluid, it is assumed that the surface fluid is sufficiently strongly bound to the solid that the probability of a bulk molecule moving into the surface layer is negligible. However, the theory below incorporates Lévy dynamics which enables the relaxation rates due to fluidfluid interactions in a surface layer, which is treated as a thin Q2D layer, to be determined for a model which allows surface spins to desorb to later return to the surface.

The derived relaxation rates are associated with the dipolar inter-molecular interaction of spin pairs due to their relative translational motion within the pore. The analysis below adopts the procedure established by Abragam [38]. The calculation is complex due to the presence of the pore boundaries which leads to an enhanced probability of close encounters of pairs of spins compared 3D bulk diffusion and yields frequency-dependent relaxation rates. For readers interested in the final result rather than the means of derivation, the key equation is the dipolar correlation function given by Eq. (13) which is transformed to the relaxation rates using Eqs. (2)-(4).

A collection of spins with a spin quantum number $I$ diffuse in a Q2D space in the presence of a static magnetic field. Nuclear spin relaxation arises due to the modulation of the dipolar interaction between pairs of $I$ spins due to their relative translational and rotational motion. $T_{1}^{-1}$ and $T_{2}^{-1}$ may be expressed in terms of the spectral density function $J(\omega)$, where $\omega$ is the Larmor frequency of a spin in the applied static field, as [38]

$$
\begin{aligned}
& T_{1}^{-1}=\frac{1}{5} \beta_{I I}[J(\omega)+4 J(2 \omega)] \\
& T_{2}^{-1}=\frac{1}{10} \beta_{I I}[3 J(0)+5 J(\omega)+2 J(2 \omega)]
\end{aligned}
$$

where $\beta_{I I}=\left(\mu_{0} / 4 \pi\right)^{2} \gamma_{I}^{4} \hbar^{2} I(I+1), \gamma_{I}$ is the proton gyromagnetic ratio and $I=\frac{1}{2}$ for protons. The spectral density function $J(\omega)$ is obtained from the Fourier transformation of the dipolar correlation function $G(t)$ defined as

$$
J(\omega)=2 \int_{0}^{\infty} G(t) \cos \omega t d t
$$

We now determine $G(t)$ for the relative translational motion of pairs of spins confined to 
a Q2D pore. The starting point is the expression [38, 39]

$$
G(t)=\frac{4 \pi}{5} \int_{\mathbb{R}^{3}} \int_{\mathbb{R}_{0}^{3}}\left[\sum_{M=-2}^{2} \frac{Y_{2 \mathrm{M}}\left(\rho_{0}, \phi_{0}, z_{0}\right) Y_{2 \mathrm{M}}^{*}(\rho, \phi, z)}{r_{0}^{3} r^{3}}\right] P\left(\mathbf{r}, t \cap \mathbf{r}_{0}\right) d^{3} \mathbf{r}_{0} d^{3} \mathbf{r}
$$

where $P\left(\mathbf{r}, t \cap \mathbf{r}_{0}\right)$ is the probability density function describing the probability distribution of pairs of spins separated by $\mathbf{r}_{0}$ at $t=0$ and by $\mathbf{r}$ at time $t$. The subscript 0 on all quantities indicates the value at $t=0$. Equation (5) incorporates powder-averaging assuming a uniform distribution of randomly-orientation pores reflecting the usual experimental practice of using powdered samples [39]. The $Y$ are the spherical harmonic functions of degree 2 expressed as functions of the cylindrical coordinates (where the asterisk superscript on the $Y$ represents the complex conjugate), $d^{3} \mathbf{r} \equiv \rho d \rho d \phi d z$ and $r^{3}=\left(\rho^{2}+z^{2}\right)^{3 / 2}$. A schematic diagram of the spin-pair space with coordinates is presented in Fig. 2.

The spin-pair coordinates $\rho$ and $z$ are independent and so

$$
P\left(\mathbf{r}, t \cap \mathbf{r}_{0}\right)=P\left(\boldsymbol{\rho}, t \cap \boldsymbol{\rho}_{0}\right) P\left(z, t \cap z_{0}\right)=P\left(\rho_{0}\right) P\left(\boldsymbol{\rho}, t \mid \boldsymbol{\rho}_{0}\right) P\left(z, t \cap z_{0}\right)
$$

where $P\left(\boldsymbol{\rho}, t \mid \boldsymbol{\rho}_{0}\right)$ is the probability density function for a pair of spins separated by an in-plane vector $\boldsymbol{\rho}$ at time $t$ given that they were separated by $\boldsymbol{\rho}_{0}$ at $t=0 . P\left(\rho_{0}\right)$ is the $a$ priori probability density function describing the probability per unit area of finding a pair of spins separated by the in-plane distance $\rho_{0} . P\left(\rho, t \mid \boldsymbol{\rho}_{0}\right)$ is obtained as a convolution of the standard solution to the diffusion equation for a point source located in an unbounded $2 \mathrm{D}$ space and may be written

$$
P\left(\boldsymbol{\rho}, t \mid \boldsymbol{\rho}_{0}\right)=\frac{e^{-\left|\boldsymbol{\rho}-\boldsymbol{\rho}_{0}\right|^{2} / 8 D t}}{8 \pi D t}
$$

where $D$ is the diffusion coefficient. $D$ is expressed in terms of the diffusion correlation time $\tau_{b}$ via Eq. (1).

The functions $P\left(\boldsymbol{\rho}, t \mid \boldsymbol{\rho}_{0}\right)$ and $P\left(z, t \cap z_{0}\right)$ are obtained as solutions to the diffusion equation and therefore model the motion of spins via a single parameter, the diffusion coefficient $D$. The theory describes the relative translational motion of spins and applies to any spinbearing fluid characterised by $D$. This paper is only concerned with translational relaxation and therefore does not take into account relaxation associated with the rotational motion of spins. The topic of rotation and other model limitations are discussed in Sec. II B. 


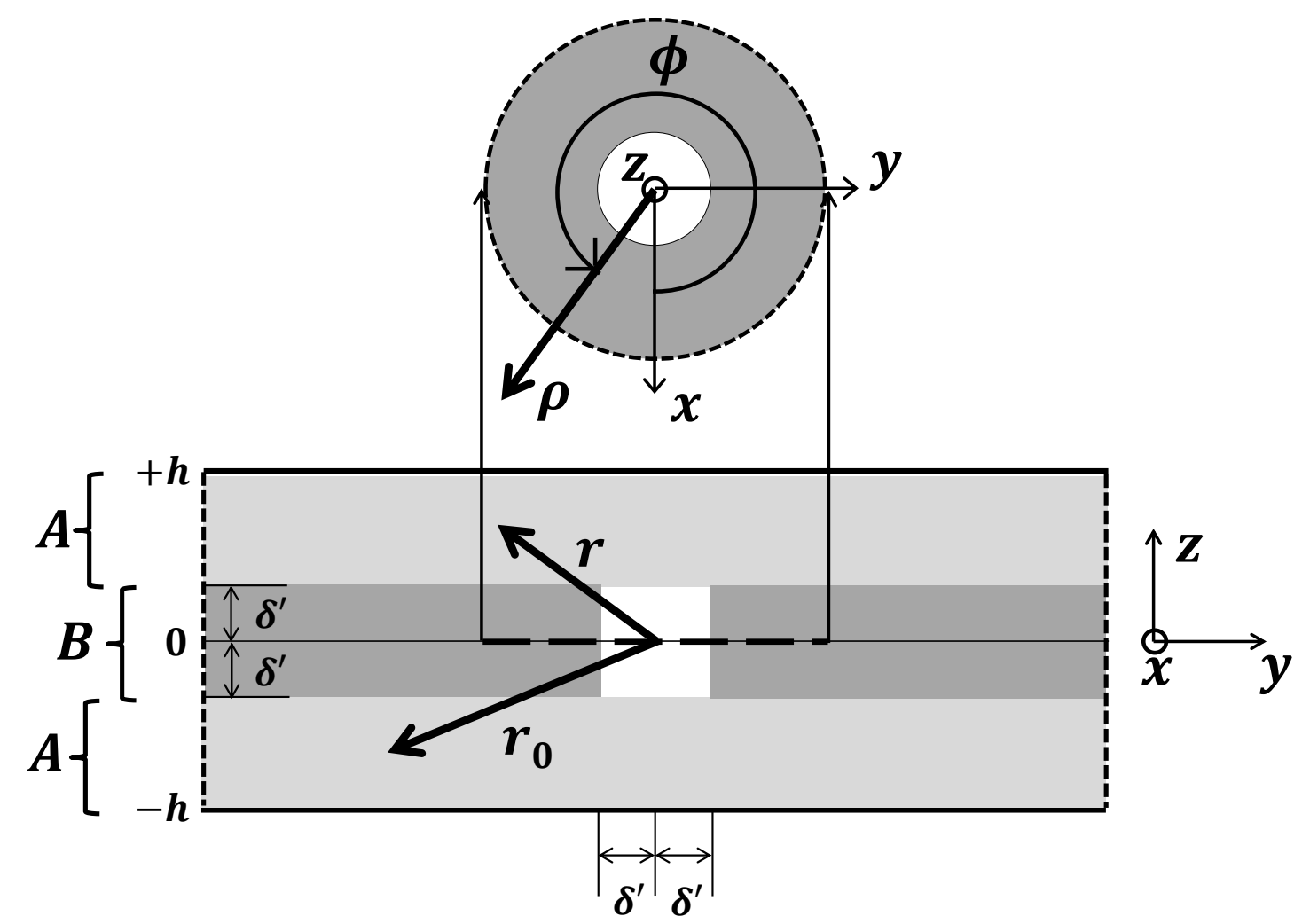

FIG. 2: A schematic diagram of the spin-pair vector space for a Q2D fluid layer unbounded in $x$ and $y$ for a pore of thickness $h$ is presented. A spin-pair vector at $t=0$ and at time $t, \mathbf{r}_{0}$ and $\mathbf{r}$ respectively, are shown with the cartesian and cylindrical coordinates of $\mathbf{r}$ illustrated. Note that

the spin-pair vector space covers the range $-h \leq z \leq+h$ for a layer of thickness $h$. The top diagram represents the $z=0$ slice as viewed from above. Dotted boundaries indicate that the space extends to infinity. Both $\mathbb{R}^{3}$ and $\mathbb{R}_{0}^{3}$ volume integrations appearing in Eq. (5) are separated into two volumes labelled $\mathrm{A}$ and $\mathrm{B}$. The exclusion volume for the integration is shown as white space and comprises a cylinder of half-height and radius $\delta^{\prime}$.

The function $P\left(\rho, t \mid \rho_{0}\right)$, as given by Eq. (7), is replaced by its well-known Fourier integral with in-plane Fourier vector variable $\mathbf{k}$ and then substituted into Eq. (5) to yield

$$
\begin{aligned}
G_{b b}(t)= & \frac{N_{\mathrm{V}}^{2 / 3}}{5 \pi} \int_{\mathbb{R}^{3}} \int_{\mathbb{R}_{0}^{3}}\left[\sum_{M=-2}^{2} \frac{Y_{2 \mathrm{M}}\left(\rho_{0}, \phi_{0}, z_{0}\right) Y_{2 \mathrm{M}}^{*}(\rho, \phi, z)}{\left(\rho_{0}^{2}+z_{0}^{2}\right)^{3 / 2}\left(\rho^{2}+z^{2}\right)^{3 / 2}}\right] \\
& \times\left\{\int e^{-2 D t k^{2}} e^{i \mathbf{k} \cdot \rho} e^{-i \mathbf{k} \cdot \rho_{0}} d^{2} \mathbf{k}\right\} P\left(z, t \cap z_{0}\right) d^{3} \mathbf{r}_{0} d^{3} \mathbf{r}
\end{aligned}
$$

noting that $P\left(\rho_{0}\right)=N_{\mathrm{V}}^{2 / 3}$ is the number of spins per unit area (where $N_{\mathrm{V}}$ is the number of spins per unit volume), $d^{2} \mathbf{k} \equiv k d k d \phi_{k}$ and $\mathbf{k}$ lies in the same plane as $\boldsymbol{\rho}$. $G_{b b}(t)$ is associated with the interaction of bulk spins with other bulk spins within the Q2D pore and 
is therefore labelled with the subscript " $b b$ ". It is noted that the Fourier integral contained in curly brackets is applicable to Fickian diffusion. This term will be adapted later to accommodate non-Fickian (Lévy) diffusion.

The exponential function $e^{i \mathbf{k} \cdot \boldsymbol{\rho}}$ in Eq. (8) is written as a Bessel function series using the Jacobi-Anger expression

$$
e^{i \mathbf{k} \cdot \boldsymbol{\rho}}=e^{i k \rho \cos \gamma}=\sum_{n=-\infty}^{\infty} i^{n} J_{n}(k \rho) e^{i n \phi} e^{-i n \phi_{k}}
$$

with $\gamma=\phi-\phi_{k}$ and where the $J_{n}$ are Bessel functions of the first kind. Using a second summation for $e^{-i \mathbf{k} \cdot \rho_{0}}$ by taking the complex conjugate of Eq. (9) and executing the integral over $\phi_{k}$, the $k$ integral in Eq. (8) reduces to

$$
2 \pi \int_{0}^{\infty} e^{-2 D t k^{2}} k d k\left[\sum_{n=-\infty}^{\infty} J_{n}(k \rho) e^{i n \phi} J_{n}\left(k \rho_{0}\right) e^{-i n \phi_{0}}\right] .
$$

This result is then inserted into Eq. (8) and the integrals over $\phi$ and $\phi_{0}$ are completed. Noting that all the integrals are zero unless $M=n$ yields

$$
\begin{aligned}
G_{b b}(t)= & \frac{2 N_{\mathrm{V}}^{2 / 3}}{5 \delta^{2}} \int_{0}^{\infty} e^{-t \kappa^{2} / 3 \tau} \kappa d \kappa \\
& \times \int_{\mathbb{R}^{2}} \int_{\mathbb{R}_{0}^{2}}\left[\sum_{M=0}^{2} h_{M}\left(\kappa, u_{0}, w_{0}\right) h_{M}(\kappa, u, w)\right] P\left(w, t \cap w_{0}\right) d u_{0} d w_{0} d u d w
\end{aligned}
$$

where $\kappa=k \delta, u=\rho / \delta$ and $w=z / \delta$ are dimensionless variables and symmetries for the $M=-1$ and $M=-2$ terms of the summation have been recognised. The $h$ functions are

$$
\begin{gathered}
h_{2}(\kappa, u, w)=\frac{1}{2} \sqrt{15 \pi} \frac{J_{2}(\kappa u) u^{3}}{\left(u^{2}+w^{2}\right)^{5 / 2}} \quad h_{1}(\kappa, u, w)=\sqrt{15 \pi} \frac{J_{1}(\kappa u) w u^{2}}{\left(u^{2}+w^{2}\right)^{5 / 2}} \\
\text { and } h_{0}(\kappa, u, w)=\frac{1}{2} \sqrt{5 \pi} \frac{J_{0}(\kappa u) u\left(2 w^{2}-u^{2}\right)}{\left(u^{2}+w^{2}\right)^{5 / 2}} .
\end{gathered}
$$

The difficulty of finding $P\left(w, t \cap w_{0}\right)$ for reflecting boundary conditions is overcome in Appendix A. Equation (A6) is then substituted into Eq. (11) followed by an evaluation of the integrals with respect to $u, u_{0}, w$ and $w_{0}$. The integrals are undertaken in Appendix B 
resulting in the following expression for the dipolar correlation function,

$$
\begin{aligned}
G_{b b}(t) & =G_{\mathrm{AA}}(t)+G_{\mathrm{AB}}(t)+G_{\mathrm{BB}}(t) \\
& =\frac{2 N_{\mathrm{V}}}{5 \delta^{3} \Delta} \int_{0}^{\infty} e^{-t \kappa^{2} / 3 \tau} \kappa\left[I_{\mathrm{AA}}(\kappa, t)+2 I_{\mathrm{AB}}(\kappa, t)+I_{\mathrm{BB}}(\kappa, t)\right] d \kappa .
\end{aligned}
$$

The upper-case subscripts A and B on the $I$ and $G$ functions do not correspond to $\ell$ or $b$ but identify the integration volumes in spin-pair space defined in Fig. 2 as detailed in Appendix B. The division of the spin-pair volume into these two regions aids mathematical tractability and also helps the interpretation of the behaviour of $G_{b b}(t)$ as discussed in Sec. IV. $I_{\mathrm{AA}}(\kappa, t)$, $I_{\mathrm{AB}}(\kappa, t)$ and $I_{\mathrm{BB}}(\kappa, t)$ are given by Eq. (B3) and the subsequent equations in Appendix B.

\begin{tabular}{|c|c|}
\hline Notation & Comment \\
\hline $\begin{array}{l}G_{b b} \\
G_{b \ell} \\
G_{\ell b} \\
G_{\ell \ell}\end{array}$ & $\begin{array}{l}\text { Lower case subscripts indicate the spin environment at } t=0 \text { for } \\
\text { spin } 1 \text { and } 2 \text { respectively, where } b \equiv \text { bulk and } \ell \equiv \text { surface layer. } \\
\text { Thus, } G_{b b} \text { is the correlation function describing the relative } \\
\text { motion of spin pairs for the case when both spin } 1 \text { and spin } 2 \\
\text { were located in the bulk at } t=0 \text {. }\end{array}$ \\
\hline $\begin{array}{l}G_{b} \\
G_{\ell}\end{array}$ & $\begin{array}{l}\text { A single lower case subscript is the sum of all contributions } \\
\text { with spin } 1 \text { in the environment indicated by the subscript. } \\
\text { Thus } G_{b}=G_{b b}+G_{b \ell} \text {. }\end{array}$ \\
\hline $\begin{array}{l}G_{\mathrm{AA}} \\
G_{\mathrm{AB}} \\
G_{\mathrm{BA}} \\
G_{\mathrm{BB}}\end{array}$ & $\begin{array}{l}\text { This notation serves mathematical convenience for the purpose } \\
\text { of executing volume integrals. Subscripts A and B refer to } \\
\text { volumes in spin-pair vector space defined in Fig. } 2 \text {. The first } \\
\text { and second subscripts refer to } t=0 \text { and } t \text { respectively. Thus, } \\
G_{\mathrm{AB}} \text { is the contribution to the correlation function describing } \\
\text { spin pair vectors in volume A at } t=0 \text { and volume B at } t \text {. }\end{array}$ \\
\hline $\begin{array}{l}G_{\mathrm{NN}} \\
G_{\mathrm{NF}} \\
G_{\mathrm{FN}} \\
G_{\mathrm{FF}}\end{array}$ & $\begin{array}{l}\text { This notation is used for the Monte Carlo simulations in } \\
\text { Sec. III. Subscript N and F refer to "near" and "far" spin pair } \\
\text { separations respectively defined by spin-pair threshold distance } \\
d \text {. Thus, } G_{\mathrm{NF}} \text { is the contribution to the MC correlation function } \\
\text { for spin pair separations such that } r_{0}<d \text { at } t=0 \text { and } r>d \text { at } \\
\text { time } t \text {. }\end{array}$ \\
\hline
\end{tabular}
For ease of reference, the notation employed for the various forms of $G$ is listed in Table I.

TABLE I: The dipolar diiffusion correlation function $G(t)$ : summary of notation and meaning

We now show the ease with which Eq.(13) can be adapted to incorporate Lévy dynamics. 
The general form of the stable symmetric Lévy distribution is given by

$$
L_{\alpha}(x)=\int e^{-|a k|^{\alpha}} e^{i k x} d k
$$

where $a$ and $\alpha$ dictate the shape of the distribution. If $\alpha=2, L_{2}(x)$ describes a gaussian distribution and Eq. (14) becomes identical to the Fourier integral in curly brackets in Eq. (8). Lévy dynamics arises if, for example, molecules execute diffusive motion on a surface with occasional excursions into a faster-moving fluid before returning to the surface. In this case, the probability density in the wings of the distribution is greater than for a gaussian. This arises when $\alpha<2$. The only analytic solution to Eq. (14) (apart from $\alpha=2$ ) arises when $\alpha=1$ which is a Cauchy distribution. The development of the theory here with the use of the Fourier integral in Eq. (8) means that the dipolar correlation function $G_{b b}(t)$ can be obtained using the expression

$$
\begin{aligned}
G_{b b}(t) & =G_{\mathrm{AA}}(t)+G_{\mathrm{AB}}(t)+G_{\mathrm{BB}}(t) \\
& =\frac{2 N_{\mathrm{V}}}{5 \delta^{3} \Delta} \int_{0}^{\infty} e^{-t \kappa^{\alpha} / 3 \tau} \kappa\left[I_{\mathrm{AA}}(\kappa, t)+2 I_{\mathrm{AB}}(\kappa, t)+I_{\mathrm{BB}}(\kappa, t)\right] d \kappa .
\end{aligned}
$$

which is identical to Eq. (13) save for the replacement of "2" by $\alpha$.

Finally, it is useful to derive analytic expressions for the dipolar correlation function at the limit $t \rightarrow 0$. The expression provides some physical insight and serves as a check on the numerical computation of Eq. (13) at short times. $G_{b b}(t \rightarrow 0)$ is evaluated by making the substitution

$$
P\left(r, t \cap \boldsymbol{r}_{0}\right)=N_{\mathrm{V}}\left(1-\frac{\left|z_{0}\right|}{h}\right) \delta\left(\boldsymbol{r}-\boldsymbol{r}_{0}\right)
$$

into Eq. (5). The ... $\mathrm{d}^{3} \boldsymbol{r}$ integral is executed for each of the spin-pair volumes A and B defined in Fig 2 before taking the limit $h \gg \delta$ to yield

$$
G_{b b}(0)=G_{\mathrm{AA}}(0)+G_{\mathrm{BB}}(0) \quad G_{\mathrm{AA}}(0)=\frac{\pi N_{\mathrm{v}}}{3 \delta^{\prime 3}} \quad G_{\mathrm{BB}}(0)=\frac{\pi N_{\mathrm{v}}}{8 \delta^{\prime 3}}(\pi+2) .
$$

It is noted that $G_{\mathrm{BB}}(0) \approx 1.9 G_{\mathrm{AA}}(0)$ and so the contribution to the correlation function due to spin pairs that lie in-plane (region B) dominates at $t=0 . G_{\mathrm{BB}}(t)$ also dominates at long times and is responsible for the evolution of the dipolar correlation function to the form $t^{-1}$ as arises for pure 2D diffusive motion in the long-time limit. 


\section{B. Limitations of the theoretical model and parameterisation}

The theoretical model describing the diffusive behaviour of spins is captured by the probability density function $P\left(\mathbf{r}, t \cap \mathbf{r}_{0}\right)$. This expression is given by Eqs. (6)-(7) and (A6). It is obvious that $P\left(\mathbf{r}, t \cap \mathbf{r}_{0}\right)$ includes significant simplifications in its description of a real fluid. For example, molecular rotation is not included [38] nor is the effect of molecular rotations on spin pair vectors connecting spins on different molecules. The $a$ priori radial spin-pair density function is assumed to be uniform so that local structure, where pairs of near-neighbor molecules have preferred orientation, is ignored. Furthermore, the probability density function $P\left(\mathbf{r}, t \cap \mathbf{r}_{0}\right)$ allows spin pairs to become arbitrarily close and to diffuse through each other.

The theoretical model disallows the close encroachment of pairs of spins by incorporating an integration exclusion volume characterised by a parameter $\delta^{\prime}$. In the present formulation the exclusion volume is a cylinder indicated in Fig. 2. This approach avoids mathematical singularities associated with a spin-pair distance of zero and acknowledges that pairs of spins cannot approach within a certain distance in the real system. Moreover, the introduction of an exclusion volume also has the advantage that $\delta^{\prime}$ can be used as a model parameter which is adjusted to best take account of model shortcomings.

Water has a ${ }^{1} \mathrm{H}-{ }^{1} \mathrm{H}$ radial density peak at the first hydration shell located at about $\delta=0.27 \mathrm{~nm}$. By contrast, the theory presented in the previous sections assumes a uniform spin density of $N_{\mathrm{V}}$ spins per unit volume in the space outside the exclusion volume. The dipolar correlation function $G_{b b}(t)$ is very sensitive to the spin-spin density at short distances. Thus, the usual practice is to treat $\delta^{\prime}$ as an adjustable model-dependent parameter chosen to compensate for the non-uniform radial density function of the real fluid. One approach is to choose $\delta^{\prime}$ so that $G_{b b}(0)$ is equal to the value obtained from MD simulations of bulk water which is approximately $40900 \mathrm{~nm}^{-6}$ [39]. This leads to $\delta^{\prime}<\delta$. For example, for a $3 \mathrm{D}$ bulk water model it was found that $\delta^{\prime}=0.7 \delta[39]$. Here, setting $G_{b b}(0)$ from Eq. (17) equal to $40900 \mathrm{~nm}^{-6}$ leads to $\delta^{\prime}=0.63 \delta$, the difference due to the different shapes of the exclusion volume (spherical in 3D, cylindrical here). However, a MD model is itself subject to approximations. The interatomic potentials may not accurately reproduce the real water structure or motions at short distances which is critical to the accurate determination of 
$G_{b b}(t)$ and thereby the relaxation rates. These shortcomings are illustrated by Calero and co-workers [40] who calculated $T_{1}$ and $T_{2}$ using four popular interatomic potential sets and an $a b$ initio approach finding that relaxation times were a factor of up to 4 times longer that the experimental value of $3.5 \mathrm{~s}$ at room temperature at a frequency of $60 \mathrm{MHz}[35]$. Furthermore, there are practical computational limits on simulation time and simulation cell size which need to be accounted for.

For this work, we adopt an alternative parameterisation approach. We acknowledge that the assumption of a uniform spin-pair probability density for $r>\delta^{\prime}$ is but one of many approximations implicit in the theoretical model used to derive Eq. (13). Consequently, we now treat $\delta^{\prime}$ simply as a single model fit parameter which is adjusted to yield $T_{1}=3.5 \mathrm{~s}$ for 3D bulk water at room temperature at a spot frequency of $60 \mathrm{MHz}$ [35]. On this basis, we adopt $\delta^{\prime}=0.243 \delta$ as the value used for all the results presented in Sec. IV and in Paper II.

It is acknowledged that this parameterisation procedure does not take into account the frequency-dependence of $T_{1}$ since $\delta^{\prime}$ is matched at a spot frequency. In particular, relaxation associated with rotational motion of molecular spins may be frequency dependent. The bulk and surface layer translational correlational times arising from analysis of data reveals these relaxation times to be short both at the surface and in the bulk. It is to be expected that rotation of molecules occurs in tandem with translation and provides an additional relaxation mechanism. Abragam [38] shows that, for a simple system characterised by an exponential correlation function with the same correlation time for rotation and translation, the relaxation rates given by the two mechanisms leads to $T_{1, \text { trans }}^{-1} \approx T_{1, \text { rot }}^{-1}$. We note the interesting molecular dynamics work of Calero and co-workers in clarifying some contributions to $T_{1}$ at a spot frequency [40]. Since the $3 \tau$ model is used to fit to the frequency-dependence of $T_{1}^{-1}$, the choice of parameterisation adopted here might impact the numerical values of the time constants $\tau_{\ell}, \tau_{d}$ and $\tau_{b}$ which emerge from the fits to experimental dispersion curves presented in Paper II.

However, the $T_{1}^{-1}$ dispersion for a bulk fluid is expected to be independent of frequency in the low-frequency range of a NMRD experiment $(\mathrm{kHz}-\mathrm{MHz})$ because the characteristic time scale for the motions of bulk fluid spins (whether due to rotations or translations) is very short, typically picoseconds or less. In practice, the bulk diffusion time constant $\tau_{b}$ is 
determined from experimental $T_{1}^{-1}$ dispersion curves at the high-frequency asymptotic limit $(>10 \mathrm{MHz})$ in Paper II. The robustness of the parameterisation procedure is evidenced in Paper II because $\tau_{b}$ obtained from fits to NMRD curves for a variety of hydrated porous systems consistently lie in the range 10-40ps ( $\approx 5$ ps for pure water) and $20-40$ ps for shale oil ( $\approx 10$ ps for pure light alkanes).

The greatest source of uncertainty associated with the parameterisation process is likely to lie with the estimation of $\tau_{\ell}$ from fits to experimental $T_{1}^{-1}$ dispersion curves because the shape of the dispersion is sensitive to $\tau_{\ell}$ over the full frequency range. The relative contributions of rotational and translational motions is sensitive to the extent to which the surface interactions restrict the movement of molecular spins. But, since the surface translations are found to have a time constant of order $\mu$ s in Paper II, relaxation due to translational motion is expected to dominate over the frequency range of a NMRD experiment.

Finally, we assume a simple liquid. This ignores problems of larger molecules where the apparent diffusivity in bulk may be time dependent (for example, for long polymer chains) and molecules with large dipoles in their own right. However, issues of spin exchange are addressed to some extent in Paper II.

\section{SIMULATION}

\section{A. The Monte Carlo model}

The MC simulation method used to model the diffusion of spins in a Q2D pore is based on the simple-hopping Ising model with spins confined to the sites of a simple cubic lattice. A schematic diagram of the model Q2D pore is presented in Fig. 3. The pore is bounded by hard walls representing the confining crystal such that the pore comprises $n$ layers and $b$ defines the lattice spacing. The simulation cell is of length $d$ in the $x$ and $y$ directions where periodic boundary conditions operate. The simulation cell contains a single layer of surface sites next to each hard-wall boundary which may contain spins that possesses different dynamical properties to those in the bulk region of the pore.

In the first instance, we consider the system in which all the spins in the pore possess the 


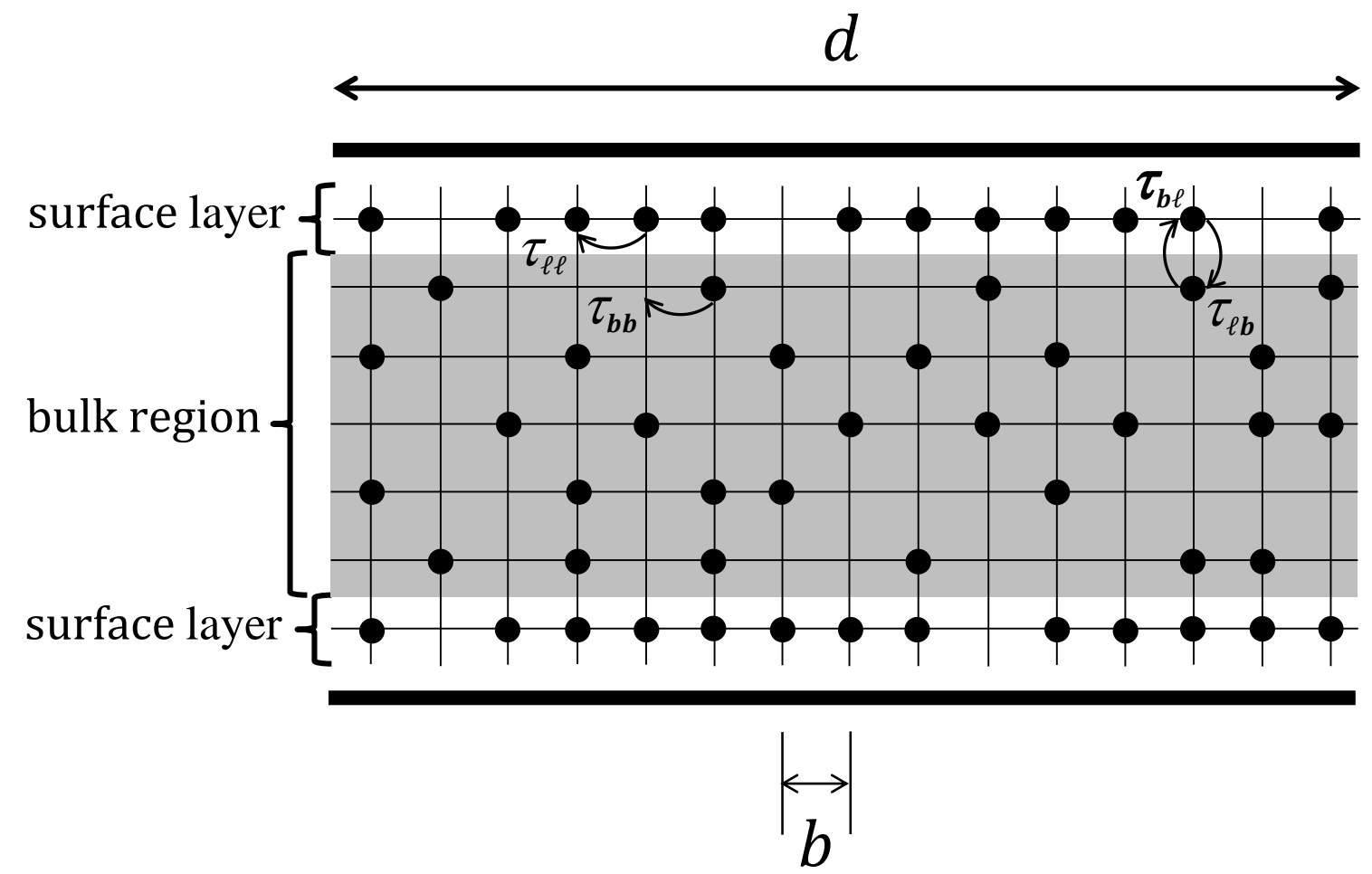

FIG. 3: The Monte Carlo model confines spins to the sites of a simple cubic lattice with a single layer of sites at each surface identified as surface sites and the remainder as bulk sites. The four mean attempt jump times which characterise the simulation are indicated for bulk-bulk, bulk-surface, surface-bulk and surface-surface attempt hops.

same dynamical properties, those pertaining to bulk water, regardless of whether they are at surface or bulk lattice sites. Here, the lattice is populated at random to a site-occupancy $c_{b}$ so that $c_{b}$ ranges from 0 to 1 . The system evolves by attempting hops at random from a probability distribution, $P_{i j}$, where $j$ is a nearest-neighbor hop event for the $i^{\text {th }}$ spin. A spin may attempt to hop to one of its six nearest-neighbor sites so that $j=1 \ldots 6$ represents one of the six possible hop events. For instance, for a particular bulk spin $i$ located next to a surface site, $j=1$ may be the event "attempt a hop to the surface site" and event $j=2$ represent "attempt a hop to a bulk site in direction $(1,0,0)$ ". For each spin $i$, an attempted hop event $j$ has an associated attempt hop rate $R_{i j}=\tau_{i j}^{-1}$. In practice of course, there are only five attempt hop types with associated rates: bulk-to-bulk $\left(\tau_{b b}^{-1}\right)$, bulk-to-surface $\left(\tau_{b \ell}^{-1}\right)$, surface-to-bulk $\left(\tau_{\ell b}^{-1}\right)$, surface to surface $\left(\tau_{\ell \ell}^{-1}\right)$ plus surface-to-wall which has a rate of 0 . 
The probability of the $i^{\text {th }}$ spin attempting a hop of type $j, P_{i j}$, is proportional to the attempt rate associated with the event $j$. The random walk simulation proceeds by generating a random number between 0 and $R$, where $R=\sum_{i j} R_{i j}$. The random number therefore identifies a spin $i$ and an attempted hop event $j$. The attempted hop is successful if the target site is not occupied. The procedure is repeated with the rate line being updated to reflect the new configuration if the attempted jump is successful. The simulation clock is incremented after each attempted hop by an amount $R^{-1}$ until the desired total simulation time has been reached.

\section{B. Parameterisation}

First, we consider the region of the pore containing bulk water. The dynamical properties of the bulk water is defined by four model parameters; the lattice parameter $b$, the mean time for a spin to make an attempted hop in the bulk, $\tau_{0}$, the fraction of lattice sites occupied by MC particles $c_{b}$ and the number of spins per MC particle, $s$. These parameters can be fixed so that the model reproduces the correct bulk water density $\rho$, diffusion coefficient $D$ and the dipolar correlation function evaluated at $t=0, G(0)$. The fourth parameter, $s$, is not strictly necessary but its introduction allows an extra degree of freedom which allows the site occupancy parameter $c_{b}$ to be chosen for the benefit of computational efficiency. A larger $c_{b}$ provides improved statistics for a given simulation size and simulation time but at the cost of increased computation time due to the large fraction of failed attempted hops. If $c_{b}$ is low, a simulation has to be executed for a long time to secure satisfactory statistics. A practical balance between the two competing demands is achieved with $c_{b} \approx 0.5$. The leaves the remaining three parameters, $s, b$ and $\tau_{0}$, to be fixed to reproduce the correct $\rho, D$ and $G(0)$.

The diffusion coefficient for particles at concentration $c$ on a $(2 \mathrm{D}$ or $3 \mathrm{D})$ discrete lattice is given by the Einstein diffusion equation [41],

$$
D=\frac{\left\langle r^{2}\right\rangle}{2 k t}=\frac{b^{2} f(c)(1-c)}{2 k \tau_{0}} .
$$

where $r$ is the distance moved by the spin in time $t$ in $k$ dimensions. $\tau_{0}$ is the mean time between attempted hops of length $b$ on a simple cubic lattice in 3D or a square lattice in 
2D. $\tau_{0}$ is related to the mean successful hop time $\tau$ by $\tau=\tau_{0} /(1-c)$. The tracer correlation factor $f(c)$ in Eq. (18) recognises that diffusion on a discrete lattice is correlated when using a site-blocking model with a finite concentration of particles. Correlated motion arises due to the enhanced probability of a particle that has just hopped moving back to its original site because, immediately after the move, the vacated site is empty whereas the alternative neighboring sites are occupied to a probability $c$. A good approximate expression for $f(c)$ is $[42]$

$$
f(c)=\left[1-\frac{2 c \theta}{(2-c)(1+\theta)}\right]^{-1}
$$

where $\theta$ takes the values $\theta_{3 \mathrm{D}}=-0.2098$ and $\theta_{2 \mathrm{D}}=-0.3634$ for simple cubic and square lattices respectively [42].

The dipolar correlation function at $t=0$ is given by

$$
G(0)=\frac{s c S_{0}}{b^{6}}
$$

where $S_{0}=\sum r_{i}^{-6}$ and $S_{0}=8.4019$ for a simple cubic lattice with a lattice constant of 1 unit. Finally, $s$ is related to the molar mass, $M$, by

$$
\frac{M}{\rho N_{A}}=\frac{b^{3}}{s c}
$$

where $N_{A}$ is Avogadro's number. The simulation parameters $s, b$ and $\tau_{0}$, presented in Table II, are determined from Eqs. (18),(19), (20) and (21) to ensure that $\rho=1000 \mathrm{~kg} / \mathrm{m}^{3}$, the experimental diffusion coefficient of water at room temperature of $2.2 \times 10^{-9} \mathrm{~m}^{2} / \mathrm{s}$ [43] and $G(0)=40900 \mathrm{~nm}^{-6}$ from molecular dynamics simulations of bulk water [39].

We now present the additional model parameters required for the simulations in which the two surface layers of water possess different properties to the bulk. The parameters that define the surface dynamics are the surface layer site occupancy $c_{\ell}$ and the layer-layer attempt hop time, $\tau_{\ell \ell}$. This allows for free choice of the surface diffusion coefficient, $D_{\ell}$, and surface spin-density. The surface spin concentration was set to $c_{\ell}=0.96$ and the attempted hop-time for surface-to-surface hops was set to $17.3 \mathrm{ps}$, chosen to give $D_{\ell} \approx 1 \times 10^{-11} \mathrm{~m}^{2} / \mathrm{s}$ which is typical of estimates of the surface water diffusion coefficient in cementitious materials [44]. The movement of spins for the surface+bulk system is now governed by the four attempt 
jump rates $R_{\ell \ell} R_{\ell b}, R_{b \ell}$ and $R_{b b}$ where $R_{b b}=\tau_{b b}^{-1}$ and $R_{\ell \ell}=\tau_{\ell \ell}^{-1}$ as described above. $R_{\ell b}$ is an adjustable parameter and $R_{b \ell}$ is determined by the detailed-balance condition $c_{b} R_{b \ell}=c_{\ell} R_{\ell b}$. Simulation parameters are presented in Table II.

\section{C. $G(t)$ from simulation}

The dipolar correlation function $G(t)$ may be obtained from the MC simulation using the expression $[39,45]$

$$
G(t)=\frac{1}{N_{i}} \sum_{i=1}^{N_{i}} \sum_{j=1}^{N_{j}} \frac{\frac{1}{2}\left(3 \cos ^{2} \psi_{i j}-1\right)}{r_{i j, 0}^{3} r_{i j}^{3}}
$$

where the summations are taken over $N_{i}$ spins in the first spin environment and $N_{j}$ spins in the second environment. $r_{i j, 0}$ and $r_{i j}$ are therefore magnitudes of the spin-pair vectors connecting the $i^{\text {th }}$ and $j^{\text {th }}$ spins at $t=0$ and time $t$ respectively. $\psi_{i j}$ represents the angle between the two vectors $\mathbf{r}_{i j, 0}$ and $\mathbf{r}_{i j}$. So, for the calculation of $G_{b \ell}(t)$, say, the summation is taken over $N_{i}$ bulk spins and $N_{j}$ surface spins.

The finite size of the simulation cell limits the maximum distances $r_{i j, 0}$ and $r_{i j}$ for use in Eq. (22) if correlation effects with neighbouring pseudo-cells are to be avoided. Spin pair separations are referred to as "near" or "far" labelled "N" and "F". "Near" spin-pairs are those that have a separation $r \leq d / 2$, and "far" spin pairs satisfy $r>d / 2$, where $d$ is the simulation cell dimension as shown in Fig. 3. Only spin-pair contributions that are in the "near" regime at $t=0$ can be included in the calculation of $G(t)$ given by Eq. (22) [48]. $G(t)$ therefore has four components written as

$$
G(t)=G_{\mathrm{NN}}(t)+G_{\mathrm{NF}}(t)+G_{\mathrm{FN}}(t)+G_{\mathrm{FF}}(t)
$$

where the first and second subscripts represents the separation of a spin-pair at $t=0$ and at time $t$ respectively. For instance, NF signifies a situation where a spin pair has $r_{0}<d / 2$ at $t=0$ and $r>d / 2$ at $t$. The notation for the various $G(t)$ functions is summarised in Table I.

The contributions $G_{\mathrm{NN}}(t)$ and $G_{\mathrm{NF}}(t)$ can be determined directly from the MC simulation using Eq. (22). The periodic boundary conditions allow the displacement of a pair of spins for which $r_{0}<d / 2$ at $t=0$ to be followed into periodic pseudo-cells allowing $r>d / 2$ at time $t$. 
Time-reversal symmetry requires that $G_{\mathrm{FN}}(t)=G_{\mathrm{NF}}(t)$ which leaves the contribution $G_{\mathrm{FF}}(t)$ which cannot be directly computed. $G_{\mathrm{FF}}(t)$ is small compared to the remaining terms in Eq. (23) at short times but is significant at longer times. Previous workers have added an analytic estimate for $G_{\mathrm{FF}}(t)$ where available [39, 45].

The different contributions to the dipolar correlation function $G(t)$ for the Q2D surface+bulk model can be calculated separately from the MC simulation. The four distinct classes of spin pairs lead to four separate correlation function components $G_{b b}, G_{b \ell}, G_{\ell b}$ and $G_{\ell \ell}$, where $G_{\mathrm{XY}}$ is calculated for the ensemble of spin pairs in which spin 1 is in region 'X' and spin 2 in region ' $\mathrm{Y}$ ' at $t=0$. It is emphasised that the ensemble that a spin identifies with is defined by its location at $t=0$ even though the spin may sample a different environment at a later time. Dipolar correlation functions and relaxation rates associated with the bulk or surface spins are defined as follows

$$
\begin{array}{cc}
G_{b}(t)=G_{b b}(t)+G_{b \ell}(t) & G_{\ell}(t)=G_{\ell \ell}(t)+G_{\ell b}(t) \\
T_{1, b}^{-1}=T_{1, b b}^{-1}+T_{1, b \ell}^{-1} & T_{1, \ell}^{-1}=T_{1, \ell \ell}^{-1}+T_{1, \ell b}^{-1} .
\end{array}
$$

$G_{b}(t)$ and $G_{\ell}(t)$ are the homonuclear powder-averaged, ensemble-average dipolar correlation functions for spins which are located in the bulk and surface regions at $t=0$ respectively. The bulk and surface regions constitute different spin environments. In the absence of exchange between surface and bulk, the separate ensembles will have different relaxation rates each of which are, in principle, observable in experiment. If the time scale for mixing is less than $T_{1}$ or $T_{2}$, a single relaxation rate is measured which is the average of the two contributions weighted by the number of spins in each environment [49].

\section{RESULTS}

\section{A. Theoretical analysis}

The results of the theoretical calculations outlined in Sec. II and the appendices are presented for the homonuclear interaction between bulk spins confined to a Q2D pore. The fluid is assumed to be bulk water of thickness $h$ with spin density $N_{\mathrm{v}}=66.6 \mathrm{~nm}^{-3}$. The model 
parameter $\delta^{\prime}=0.243 \delta$ where the elementary distance is $\delta=0.27 \mathrm{~nm}$ as described in Sec. II. The diffusion coefficient of water is $0.0022 \mathrm{~nm}^{2} \mathrm{ps}^{-1}$ for pure water at $25^{\circ} \mathrm{C}$ which leads to the diffusion correlation time $\tau=5.3$ ps.

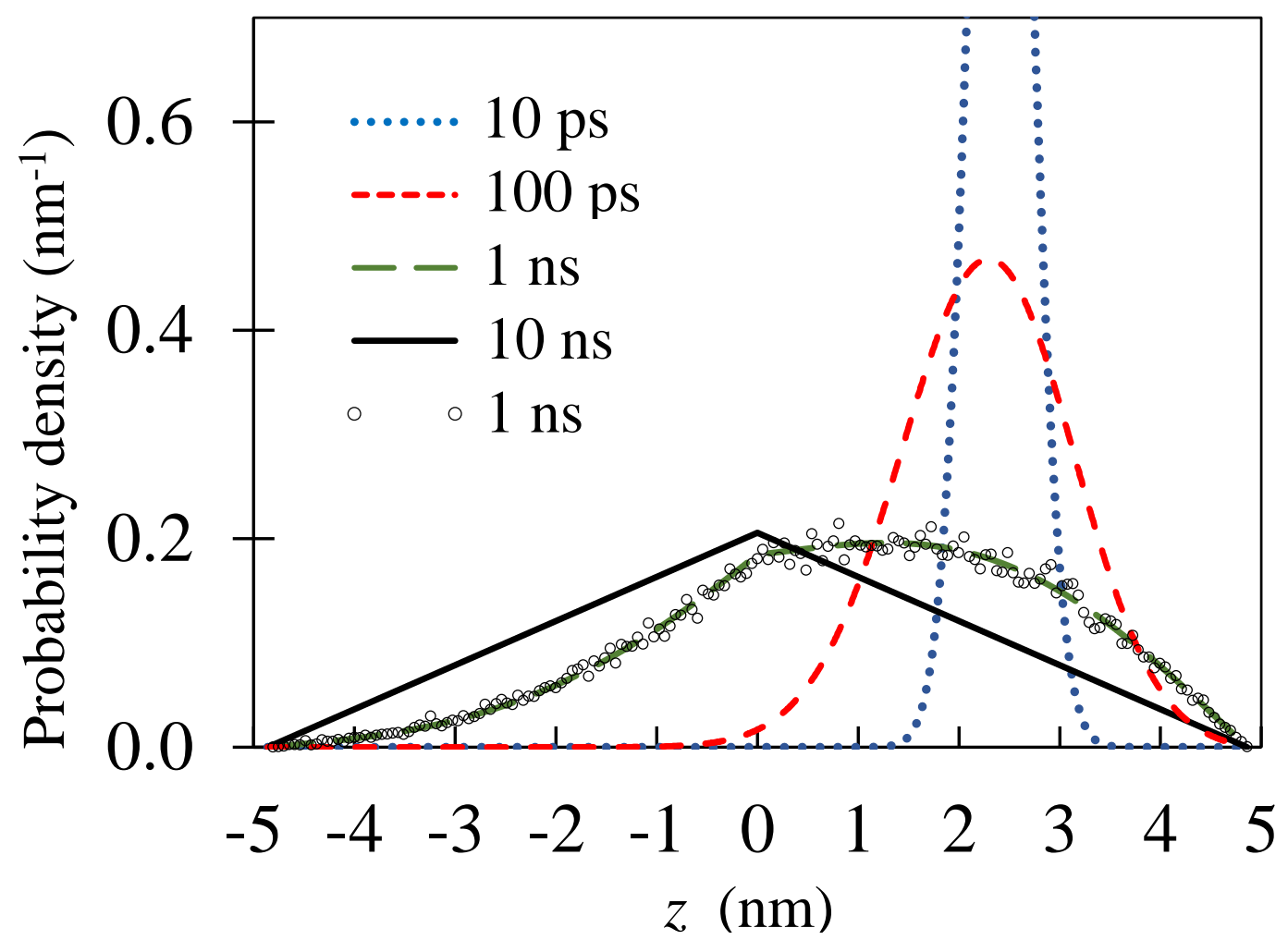

FIG. 4: The figure shows the evolution in time of the spin-pair probability density function $P\left(z, t \mid z_{0}\right)$ for the case $z_{0}=+2.5 \mathrm{~nm}$ and $h=5 \mathrm{~nm}$.

Results for the conditional probability density function $P\left(z, t \mid z_{0}\right)$, the dipolar correlation function $G_{b b}(t)$, and the spin-lattice relaxation rate $T_{1, b b}^{-1}$ are presented. Recall that $z$ is a spin-pair distance in the direction across the pore and extends from $-h$ to $+h$ (see Fig. 2). An example of the conditional probability density function $P\left(z, t \mid z_{0}\right)$ describing the probability of a spin pair being separated by a distance $z$ at time $t$, given that the pair were separated by $z_{0}$ at $t=0$, is presented in Fig. 4. This is computed from Eqs. (A4-A5). The computation of Eq. (A4) is undertaken for a pore of thickness $h=5 \mathrm{~nm}$ assuming $z_{0}=+2.5 \mathrm{~nm}$. Thus, at $t=0, P\left(z, t \mid z_{0}\right)$ is a delta-function located at $z_{0}=+2.5 \mathrm{~nm}$. After $10 \mathrm{ps}$ and $100 \mathrm{ps,}$ the distribution is approximately gaussian as expected from the standard solution to the diffusion equation. After $1 \mathrm{~ns}$, the spin-pair distribution has become asymmetric as the individual spins reflect from the hard-wall pore boundaries. The development of a gradient 
discontinuity is apparent at $z=0$. A simple $1 \mathrm{D} \mathrm{MC}$ simulation confirms the accuracy of the calculation at $1 \mathrm{~ns}$. After $10 \mathrm{~ns}$, the spin-pair has lost memory of its original separation and forms the classic triangular distribution given by $P\left(z_{0}\right)$ presented as Eq. (A1).

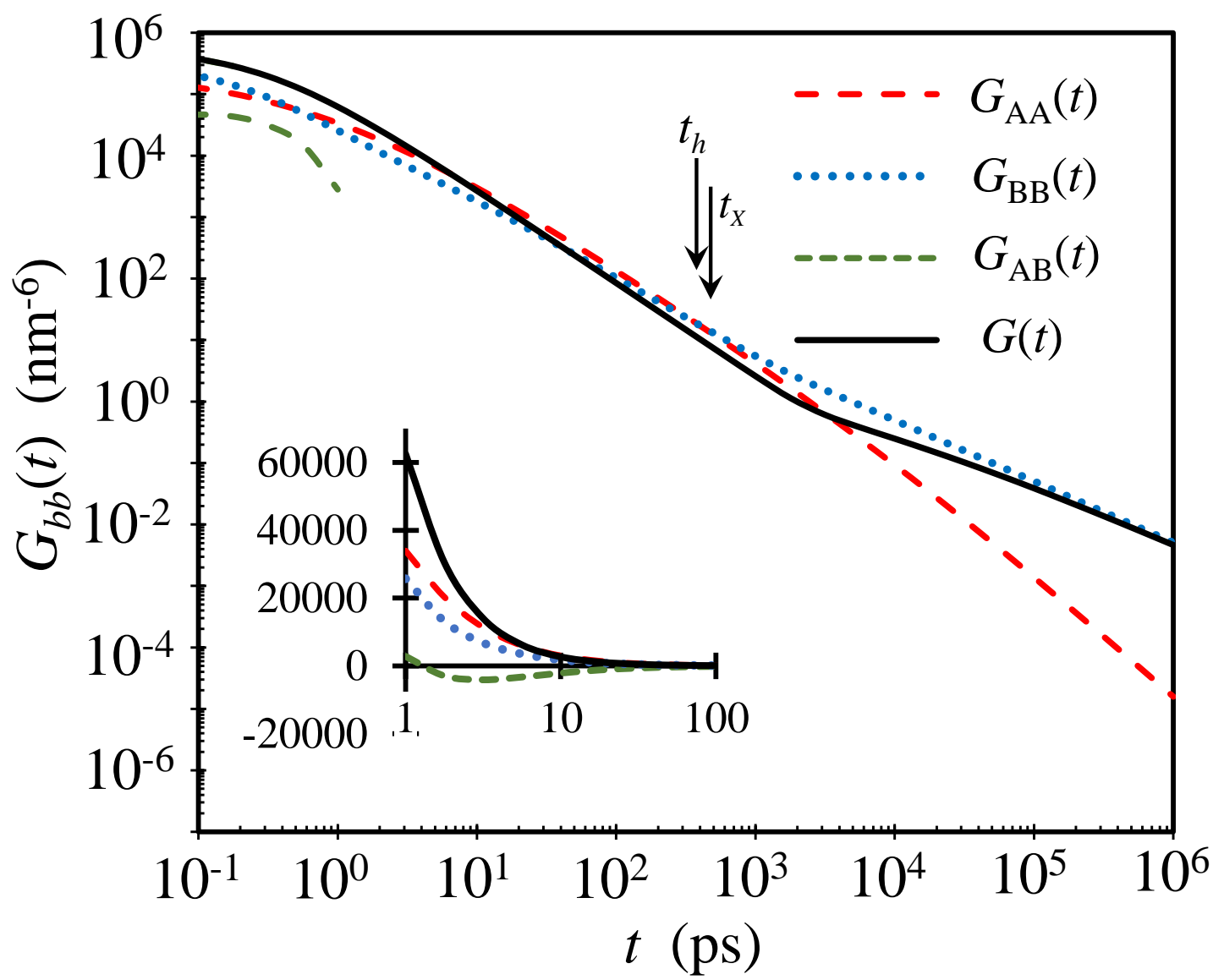

FIG. 5: The dipolar correlation function $G_{b b}(t)$ (solid line) is plotted as a function of $t$ for a water-filled planar pore of thickness $h=5 \mathrm{~nm}$ and spin diffusion correlation time $\tau_{b}=5.3 \mathrm{ps}$. The contributions $G_{\mathrm{AA}}(t)$ (long dash, red online) and $G_{\mathrm{BB}}(t)$ (dotted line, blue online) are shown. $G_{\mathrm{AB}}$ (short dash, green online) is negative as illustrated in the insert. Here A and B refer to spin-pair vectors which are "out of plane" and "in plane" respectively (see Table I). $t_{\mathrm{x}}$ is the time at which $G_{\mathrm{BB}}=G_{\mathrm{AA}}$ and $t_{h}$ is the average time for a spin to move a distance $h$.

Figure 5 presents the bulk-bulk dipolar correlation function $G_{b b}(t)$ for a pore of thickness $5 \mathrm{~nm} . G_{b b}(t)$ is computed using Eq. (13) with a spin diffusion correlation time $\tau_{b}=5.3$ ps for bulk water at room temperature. $G_{b b}(t)$ has the form first identified in Ref. [39], that is, the correlation function behaves approximately as for a 3D system of spins before making a change of gradient tending to $t^{-1}$ at long times, characteristic of $2 \mathrm{D}$ diffusion. Fig. 5 demonstrates why the bulk-bulk correlation function $G_{b b}(t)$ takes this form. The contributions to $G_{b b}(t)$ for spin pair vectors in region A or region B at both $t=0$ and at time $t, G_{\mathrm{AA}}(t)$ or 
$G_{\mathrm{BB}}(t)$ respectively, are also plotted in the figure. Recall that $G_{\mathrm{AA}}(t)$ is the contribution to $G_{b b}(t)$ due to spin-pair vectors that lie out-of-plane (such that $\delta^{\prime}<|z|<h$ ) both at $t=0$ and at time $t$ as illustrated in Fig. 2. The spin-pair vectors which lie in-plane $\left(|z|<\delta^{\prime}\right)$ at $t=0$ and at time $t$ are captured by $G_{\mathrm{BB}}(t) . G_{b b}(t)$ is dominated by the evolution of in-plane spin-pair vectors at very short times and at longer times and by out-of-plane spin-pair vectors at intermediate times. The crossover arises when $G_{\mathrm{AA}}(t)=G_{\mathrm{BB}}(t)$ indicated by $t_{\mathrm{x}}$ in the figure.

The inset figure in Fig. 5 shows the correlation functions on a linear-log plot over the time scale 1-100ps. This shows that $G_{\mathrm{AB}}(t)$, which captures the contribution of in-plane spin-pair vectors moving out-of-plane and vice versa, provides a negative contribution to $G_{b b}(t)$. The mean time, $t_{h}=h^{2} / 6 D$, for a spin to move a distance $h$ in the direction perpendicular to the pore surface (which is a measure of the time for the pore boundaries to impact the spin-pair probability density function) is also shown. It is evident that $t_{\mathrm{x}} \approx t_{h}$ but that neither indicates the time of the change in gradient indicating the transition from $3 \mathrm{D}$ to $2 \mathrm{D}$ behaviour which occurs at about $10 t_{\mathrm{x}}$. This is because the contribution $G_{\mathrm{AB}}(t)$ continues to provide a significant negative contribution to the correlation function in this regime.

Figure 6 presents $G_{b b}(t)$ for a range of pore thickness from $1 \mathrm{~nm}$ to $50 \mathrm{~nm}$. All curves tend to $G(0)$ as given by Eq. (17) as $t \rightarrow 0$. The correlation function for unconfined 3D bulk water takes the form $G_{b b}(t) \propto t^{-3 / 2}$ at long times and the line labelled $t^{-3 / 2}$ in Fig. 6 shows the pristine $3 \mathrm{D}$-like behaviour over a time which is dependent on the pore thickness.

An analogous system to the Q2D plane has been explored by Grebenkov et al [46]. These workers investigated the motion of spins confined to a thin layer at the surface of a sphere (as a model protein). It was argued that two long-time behaviours were important which are related to the movement of spins within the layer either parallel to the surface of the sphere or in the transverse (radial) direction. Without executing a full calculation of the relative motion of pairs of diffusing spins confined to a spherical shell, these authors argued that the long-time behaviour of the dipolar correlation function should be proportional to the "probability of return" of a spin. In the context of the relative motion of pairs of spins, the "probability of return" refers to the probability that a pair of spins in close proximity at $t=0$ return to close proximity at a later (long) time $t$. Grebenkov et al argued that the transverse contribution to the dipolar correlation function was 3D-like and decayed as 


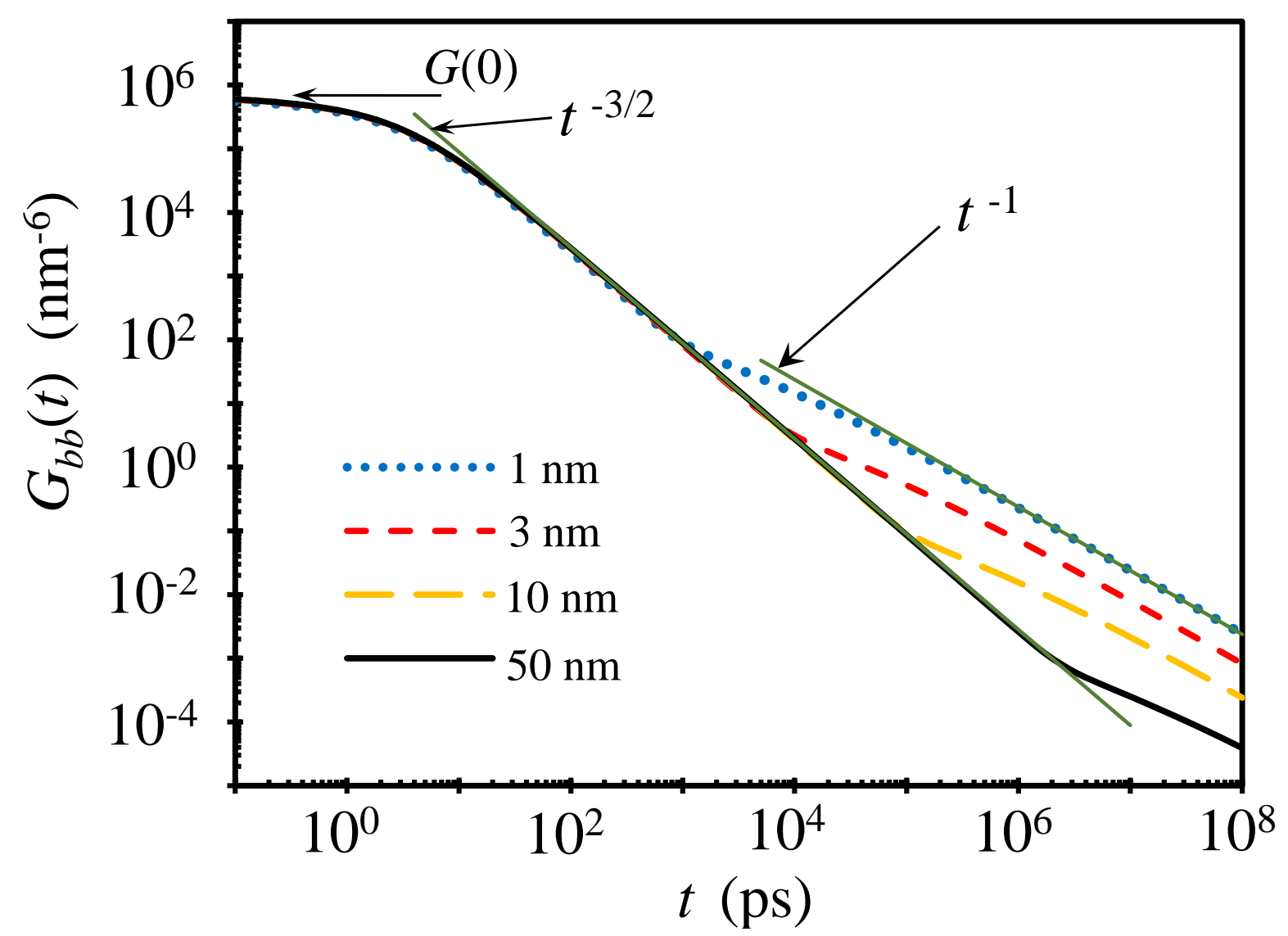

FIG. 6: The figure presents $G_{b b}(t)$ for pore thicknesses $h=1,3,10$ and $50 \mathrm{~nm}$. The spin diffusion correlation time $\tau_{b}$ is $5.3 \mathrm{ps}$.

$t^{-3 / 2}$ whilst the parallel component decayed as $t^{-1}$, as for a system with all spins confined to $2 \mathrm{D}$. The results presented here are in agreement with these conclusions, as illustrated in both Fig. 5 and Fig. 6, insofar as the 3D-like $t^{-3 / 2}$ and 2D-like $t^{-1}$ behaviours are clearly demonstrated. Our results show that the relative motion of spin pairs is 3D-like until the impact of the pore boundaries is felt. Once a significant fraction of spins have experienced reflection from the pore boundaries, the system evolves to 2D-like behaviour.

The transition time $t_{\mathrm{x}}$ is dependent on the pore thickness as explored in Fig. 7. Here $t_{\mathrm{X}}$ is estimated as the time when the gradient of $G_{b b}(t)$ is changing most rapidly. Figure 7 shows that $t_{\mathrm{x}} \propto h^{2}$, consistent with earlier observations [39] and also with the conclusions of Grebenkov at al [46]. Note that the long-time $t^{-1}$ behaviour of the bulk-bulk correlation 


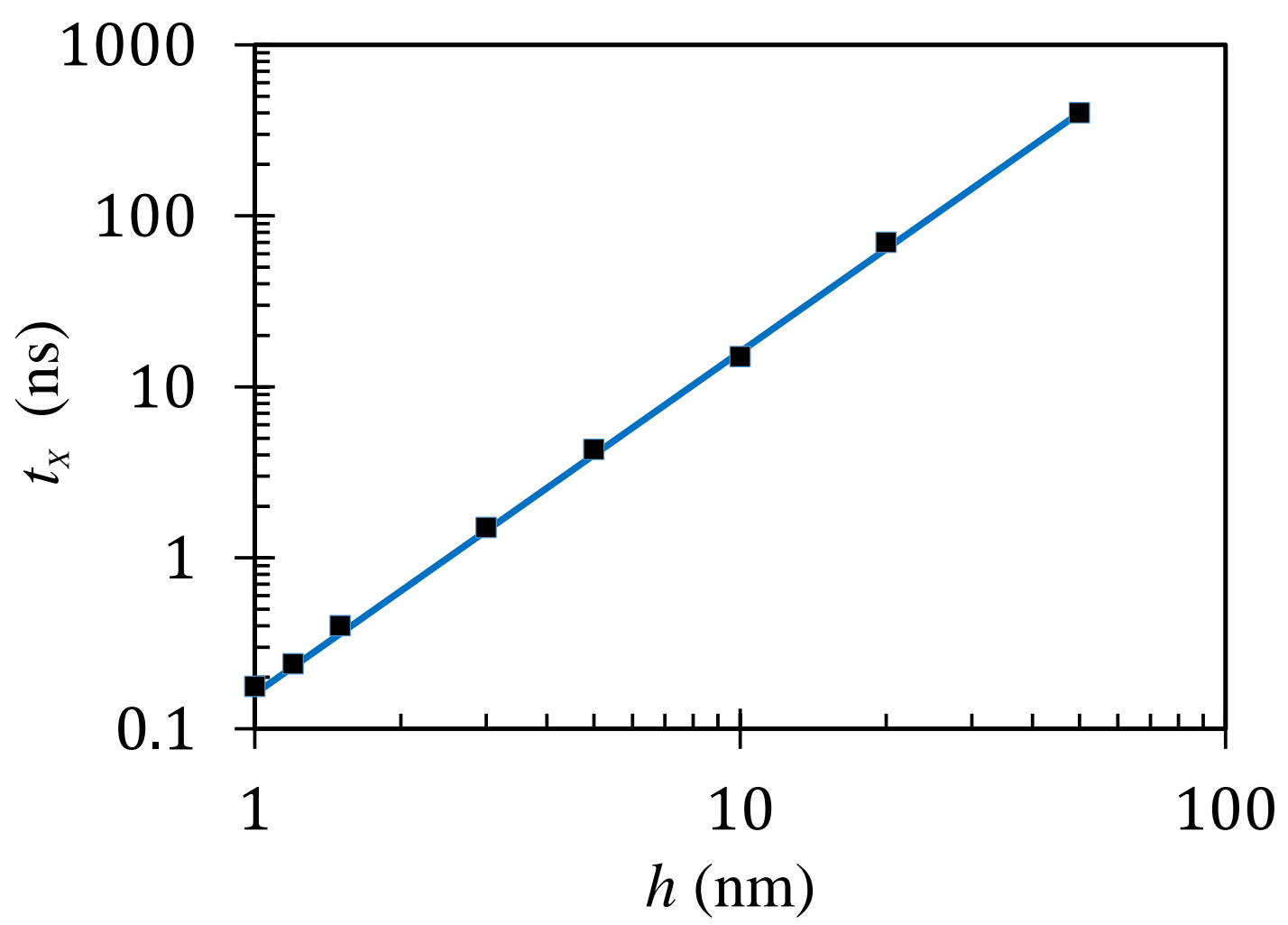

FIG. 7: The figure shows the time, $t_{\mathrm{x}}$, for the correlation function $G_{b b}(t)$ to make the 3D-2D transition as a function of pore thickness $h$ The line has a gradient of 2 .

function $G_{b b}(t)$ is only seen after $t \approx 10 \mu$ s in a $50 \mathrm{~nm}$ pore despite the short (5.3 ps) diffusion correlation time for water.

The spin-lattice relaxation rate $T_{1, b b}^{-1}$ is obtained from the Fourier transformation of $G_{b b}(t)$ via Eqs. (2) and (4). Figure 8 shows the pore-size dependency of $T_{1, b b}^{-1}$ as a function of frequency over the frequency range 0.01-100 MHz. The qualitative behaviour is consistent with a similar approximate calculation by Korb et al for a Q2D layer containing mobile spins and paramagnetic impurities [3]. $T_{1, b b}^{-1}$ is found to be approximately constant for pore thickness $h>10 \mathrm{~nm}$. The value of $T_{1}$ for bulk water at room temperature and at a frequency of $60 \mathrm{MHz}$ is about $3.5 \mathrm{~s}$ [35]. Fig. 8 shows that, for pores less than about $3 \mathrm{~nm}, T_{1, b b}^{-1}$ increases with decreasing frequency.

The final analysis examines the dependence of $T_{1, b b}^{-1}$ on the bulk water diffusion correlation time $\tau_{b}$. Although $\tau_{b}=5.3 \mathrm{ps}$ for pure water at room temperature [35], water in porous 


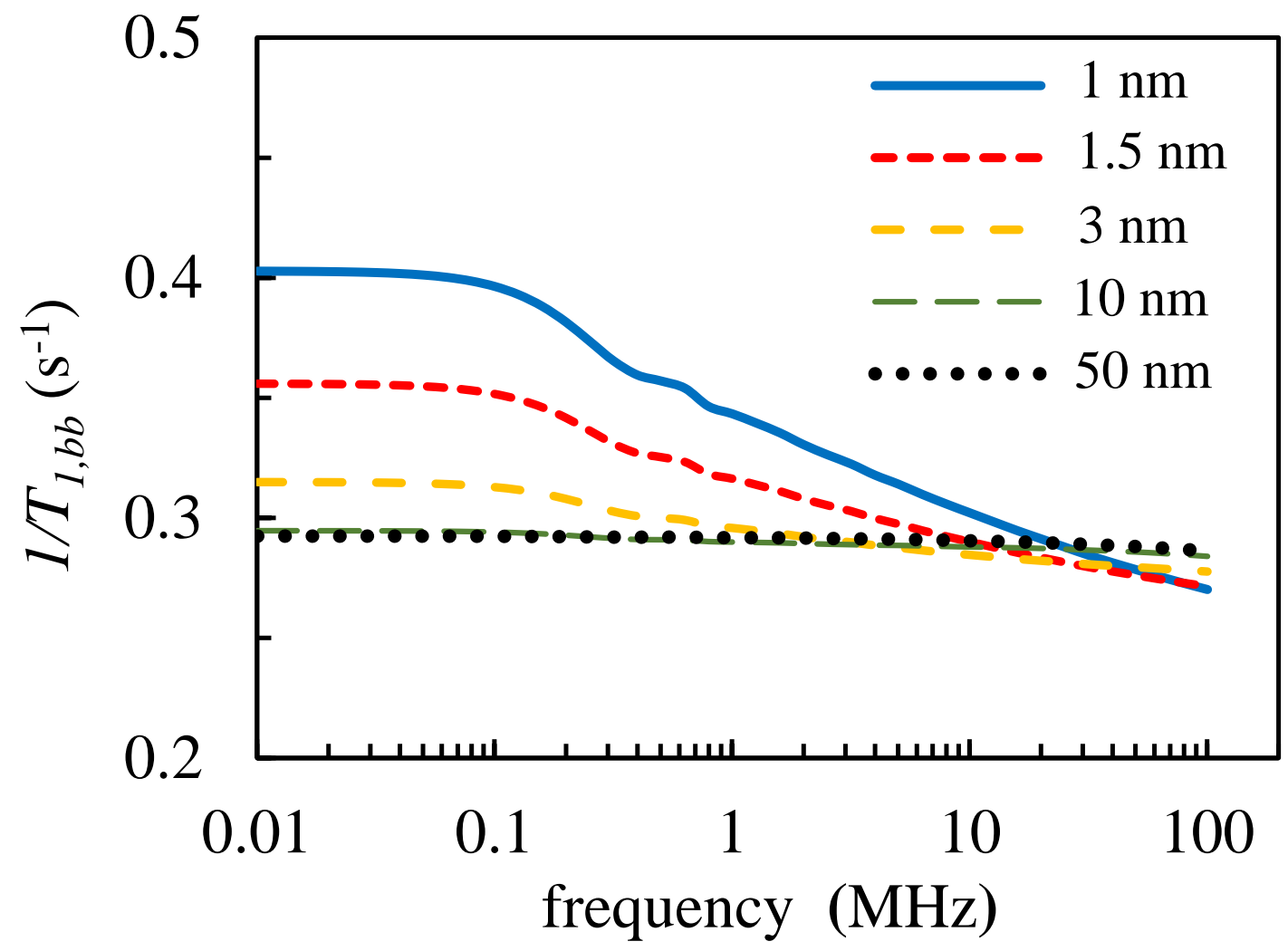

FIG. 8: The spin-lattice relaxation rate $T_{1, b b}^{-1}$ for pore thickness in the range $1 \mathrm{~nm}$ to $50 \mathrm{~nm}$ at typical experimental frequencies are presented. The spin diffusion correlation time $\tau_{b}$ is $5.3 \mathrm{ps}$. $T_{1, b b}^{-1}$ is almost independent of frequency except for pores with $h \lesssim 3 \mathrm{~nm}$.

media will contain dissolved ions which will reduce the average water diffusion coefficient or, equivalently, increase the effective $\tau_{b}$. Figure 9 presents $T_{1, b b}^{-1}$ as a function of $\tau_{b}$. $T_{1, b b}^{-1}$ is found to be proportional to $\tau_{b}$ over the range 5-40 ps.

Figure 9 enables a direct measure of the diffusion coefficient of the bulk fluid from the high-frequency limit of $T_{1, b b}^{-1}$ in porous systems in which the relaxation is not dominated by paramagnetic impurities. For example, Korb [10] presents results for plaster paste which shows that $T_{1}^{-1} \approx 1.1 \mathrm{~s}^{-1}$ at high frequency. If it is assumed that $T_{1}^{-1} \approx T_{1, b b}^{-1}$ this would imply $\tau_{b} \approx 21.5$ ps corresponding to $D \approx 5.7 \times 10^{-10} \mathrm{~m}^{2} / \mathrm{s}$. A more detailed analysis including other contributions in Paper II yields $\tau_{b} \approx 17$ ps. Thus this result allows the diffusion coefficient of the bulk fluid to be obtained to good accuracy from the high-frequency dispersion limit without the need for additional experimentation. 


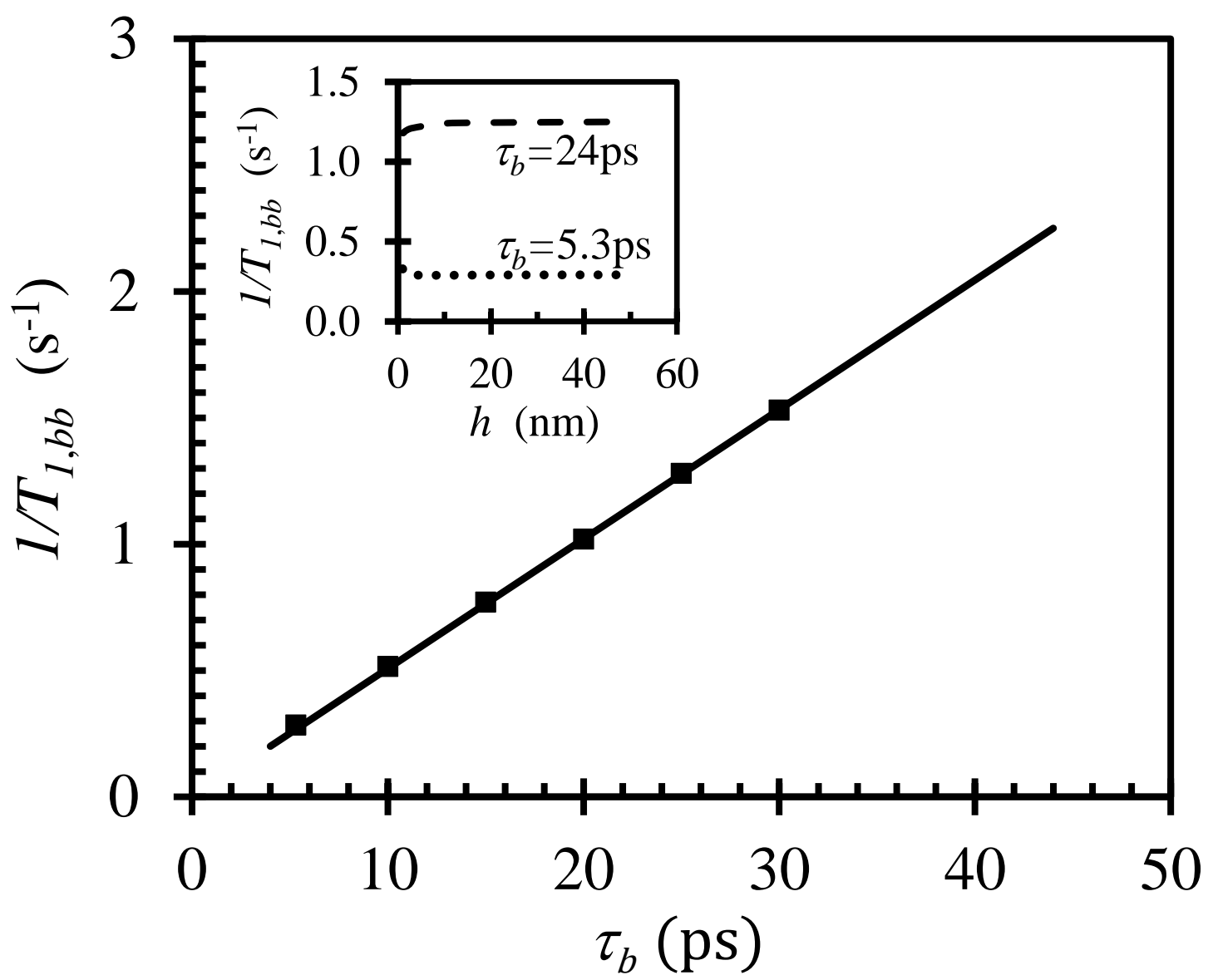

FIG. 9: The spin-lattice relaxation rate $T_{1, b b}^{-1}$ is presented as a function of the bulk diffusion correlation time $\tau_{b}$ for a pore thickness of $5 \mathrm{~nm}$. The inset shows $T_{1, b b}^{-1}$ as a function of bulk fluid layer thickness $h$ evaluated at $20 \mathrm{MHz}$.

\section{B. MC simulations}

We now present the results of MC simulations for Q2D pores which include surface water represented as single layers adjacent to the pore surfaces, as illustrated in Fig. 3. The remainder of the pore contains water with bulk-like properties. Results for $T_{1, b b}^{-1}$ as a function of pore thickness have already been published [39]. For the present simulations, the pore comprised 10 layers $(1.89 \mathrm{~nm})$ so that the thickness of the bulk water component is about $1.5 \mathrm{~nm}$. Simulation parameters are presented in Table II.

The diffusive properties of the spins in the surface layer are characterised by two time parameters, the surface-surface attempt hop time $\tau_{\ell \ell}$ and desorption time $\tau_{d}$. $\tau_{\ell \ell}$ governs 


\begin{tabular}{lll}
\hline \hline MC simulation parameter & Symbol & Value \\
\hline Lattice constant & $b$ & $0.189 \mathrm{~nm}$ \\
Attempt/actual bulk-bulk hop time & $\tau_{b b} / \tau_{\text {bb-act }}$ & $1.23 \mathrm{ps} / 2.23 \mathrm{ps}$ \\
Attempt/actual surface-surface hop time & $\tau_{\ell \ell} / \tau_{\ell \ell-\text { act }}$ & $17.3 \mathrm{ps} / 0.43 \mathrm{~ns}$ \\
Bulk spin concentration & $c_{b}$ & 0.45 \\
Surface spin concentration & $c_{\ell}$ & 0.96 \\
Spin concentration scaling factor & $s$ & 0.49 \\
Cell dimensions & $d \times d \times h$ & $3.78 \mathrm{~nm} \times 3.78 \mathrm{~nm} \times 1.89 \mathrm{~nm}$ \\
Number of spins & $n_{\text {spin }}$ & $\approx 2200$ \\
Surface residency times & $\tau_{d}$ & $0.5 \mathrm{~ns}, 3.8 \mathrm{~ns}, 18.5 \mathrm{~ns}$, \\
& & $54.5 \mathrm{~ns}, 178.2 \mathrm{~ns}, 60+\mu \mathrm{s}$ \\
Total simulation runtime & $t_{\text {tot }}$ & $0.5 \mu \mathrm{s}$ \\
\hline \hline
\end{tabular}

TABLE II: MC simulations: summary of parameters

the rate of hopping to neighbouring sites within the surface layer and therefore determines the diffusion coefficient of spins at the surface. The diffusion coefficient for the spins in the surface layer was set to $D \approx 10^{-11} \mathrm{~m}^{2} / \mathrm{s}$ which translates to $\tau_{\ell \ell}=0.43 \mathrm{~ns}$, consistent the interpretation of experimental dispersion curves using a Korb model $[3,4,6,10,11,15]$ and, critically here, short enough to be accessible to $\mathrm{MC}$ simulations.

The characteristic desorption time $\tau_{d}$ is a measure of the surface affinity. Within the MC model, the exchange of spins between the surface and bulk environments is governed by the rate parameter $\tau_{\ell b}$. For a chosen $\tau_{\ell b}$, the desorption time $\tau_{d}$ is calculated by counting the number of spins on the surface at time $t$. The fraction of spins remaining on the surface (spins desorbing and subsequently returning to the surface are excluded from the calculation) is fit to $A e^{-t / \tau_{d}}$ to yield $\tau_{d}$. Simulations were completed for $\tau_{d}$ equal to $0.5 \mathrm{~ns}, 3.8 \mathrm{~ns}, 18.5 \mathrm{~ns}$, $54.5 \mathrm{~ns}, 178 \mathrm{~ns}$ and $60+\mu \mathrm{s}$. The shortest times are consistent with MD simulation results for water on silicate surfaces [39] and the longest times consistent with values emerging from oil/water in rocks [11], granular silicate material, limestone cores [6], cementitious materials $[8,9,15]$ when fit to a Korb model and consistent with values emerging from our re-analysis of a range of systems in Paper II. The MC simulation labelled $\tau_{d}=60+\mu$ s is one in which there was no detectable desorption during the time scale of the simulation.

Figure 10 presents the results for the dipolar correlation function for spin pairs where 


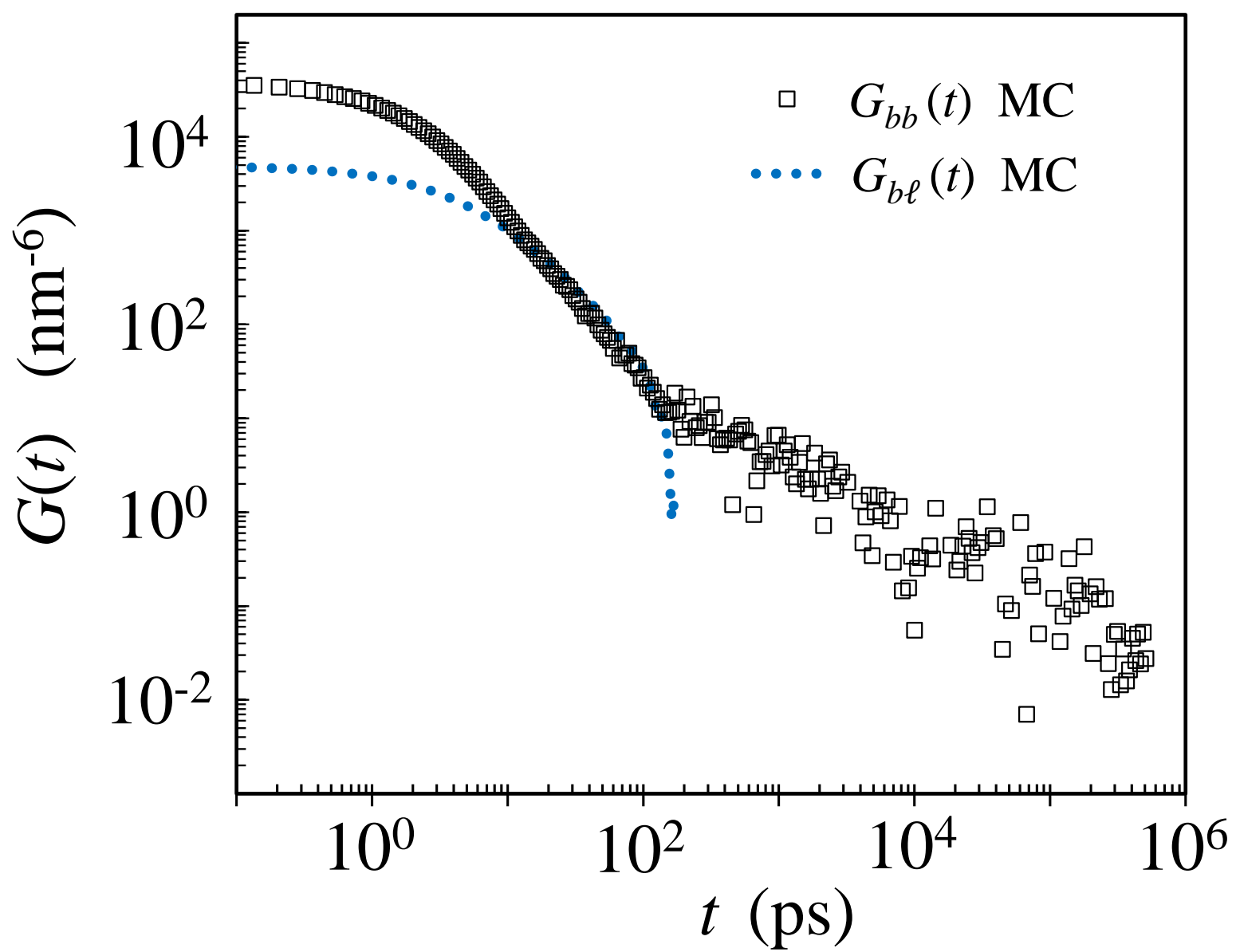

FIG. 10: The MC results for $G_{b b}(t)$ and $G_{b \ell}(t)$ are presented for a pore of thickness $h=1.89 \mathrm{~nm}$ with a surface desorption time $\tau_{d}=0.5 \mathrm{~ns}$.

both spins were in the bulk at $t=0, G_{b b}(t)$, and where spin 2 was in the surface layer at $t=0, G_{b \ell}(t)$. The surface desorption time is $\tau_{d}=0.5 \mathrm{~ns}$ in this example. Figure 10 shows that $G_{b \ell}(t)$ executes a rapid decorrelation associated with spins moving between the bulk and surface layer environments. Hops between the surface and bulk can lead to an angular change in spin pair vectors which produces a negative contribution to the correlation function via Eq. (22), in the same manner as for $G_{\mathrm{AB}}(t)$ presented in Fig, 5. The transition to $2 \mathrm{D}$ is seen as an abrupt change of gradient at about $100 \mathrm{ps}$. The form of $G_{b b}(t)$ is consistent with the theoretical calculations presented earlier. The Fourier transformation of $G_{b}(t)=G_{b b}(t)+G_{b \ell}(t)$ finds $T_{1, b}^{-1}=0.13 \pm 0.02 \mathrm{~s}^{-1} . \quad T_{1, b}^{-1}$ is frequency-independent for $f<100 \mathrm{MHz}$. Note that the MC simulation is not parameterised to obtain the experimental relaxation rate for water. 


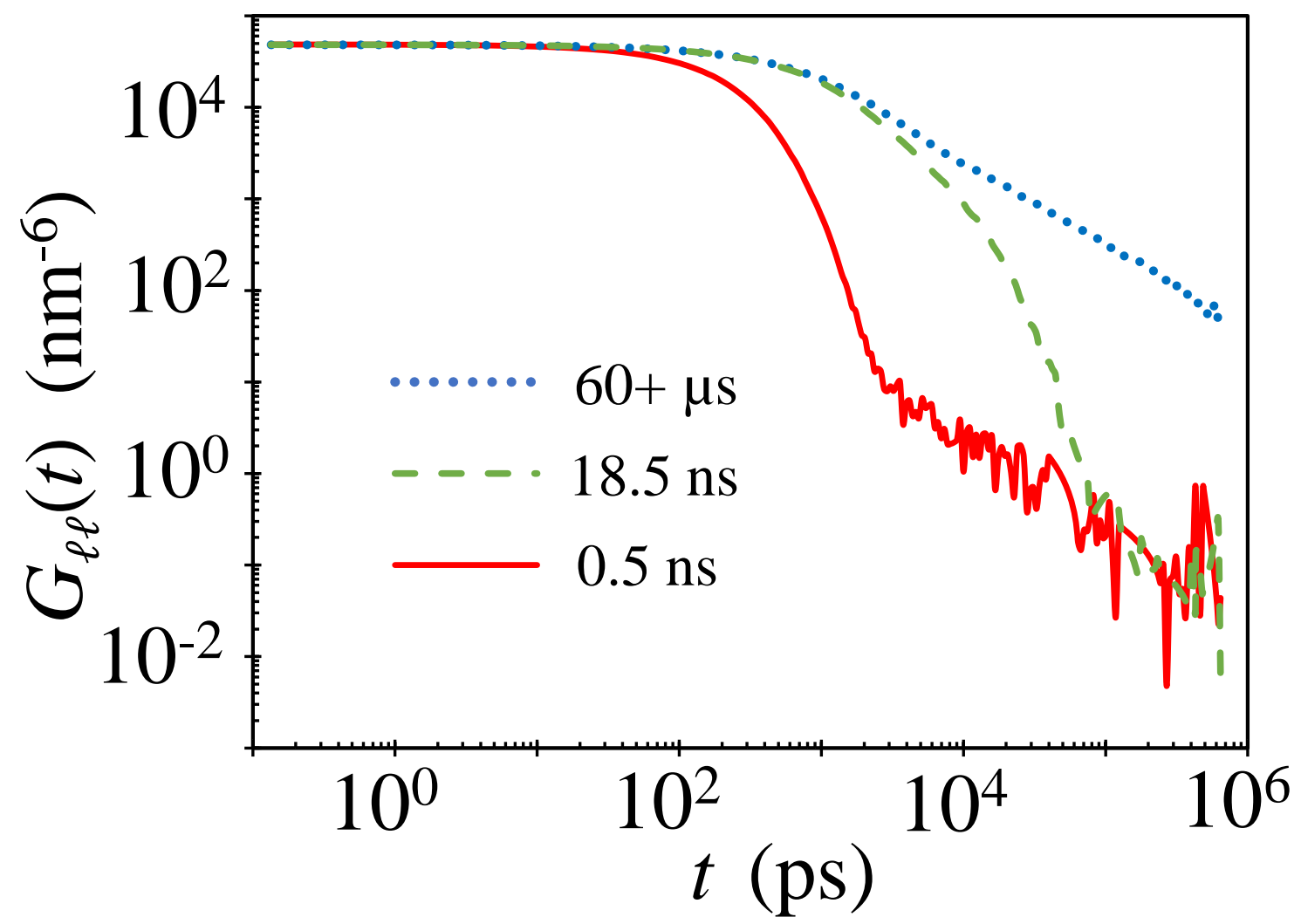

FIG. 11: The MC results for $G_{\ell \ell}(t)$ are presented for a pore of thickness $h=1.89 \mathrm{~nm}$ with surface desorption times of $\tau_{d}=0.5 \mathrm{~ns}, 18.5 \mathrm{~ns}$ and $60+\mu s$.

We now consider the contribution to the relaxation rate due to the spins contained in the surface layers both at $t=0$ and at time $t$. Figure 11 presents the results for $G_{\ell \ell}(t)$. $G_{\ell b}(t)$ (not shown) is identical in form to $G_{b \ell}(t)$ with the magnitude scaled by the ratio $N_{b} / N_{\ell}$. The results for $G_{\ell \ell}(t)$ are strongly influenced by the desorption correlation time $\tau_{d}$. The data for $\tau_{d}=60+\mu s$, in which there is no exchange between the surface and bulk spins, show the classic 2D dipolar correlation function, namely $G_{\ell \ell}(t) \propto t^{-1}$ for $t \gtrsim 1 \mathrm{~ns}$. For the shortest desorption time, $\tau_{d}=0.5 \mathrm{~ns}$, the surface spins do not have the opportunity to diffuse within the surface before the desorption of spins occurs. The correlation function starts to decay at $t \approx \tau_{d}$ due to the loss of spins from the surface. The curve then displays an abrupt change in gradient at $t \gtrsim 5 \mathrm{~ns}$ which is associated with surface spins that desorb to the bulk and then later return to the surface. This constitutes non-Fickian, or Lévy, diffusion which is characterised by $G_{\ell \ell}(t) \propto t^{-n}$ at long times where $n<1$. The measured gradient yields $n=0.9$, but the MC simulations exclude the contribution from spin pairs separated 


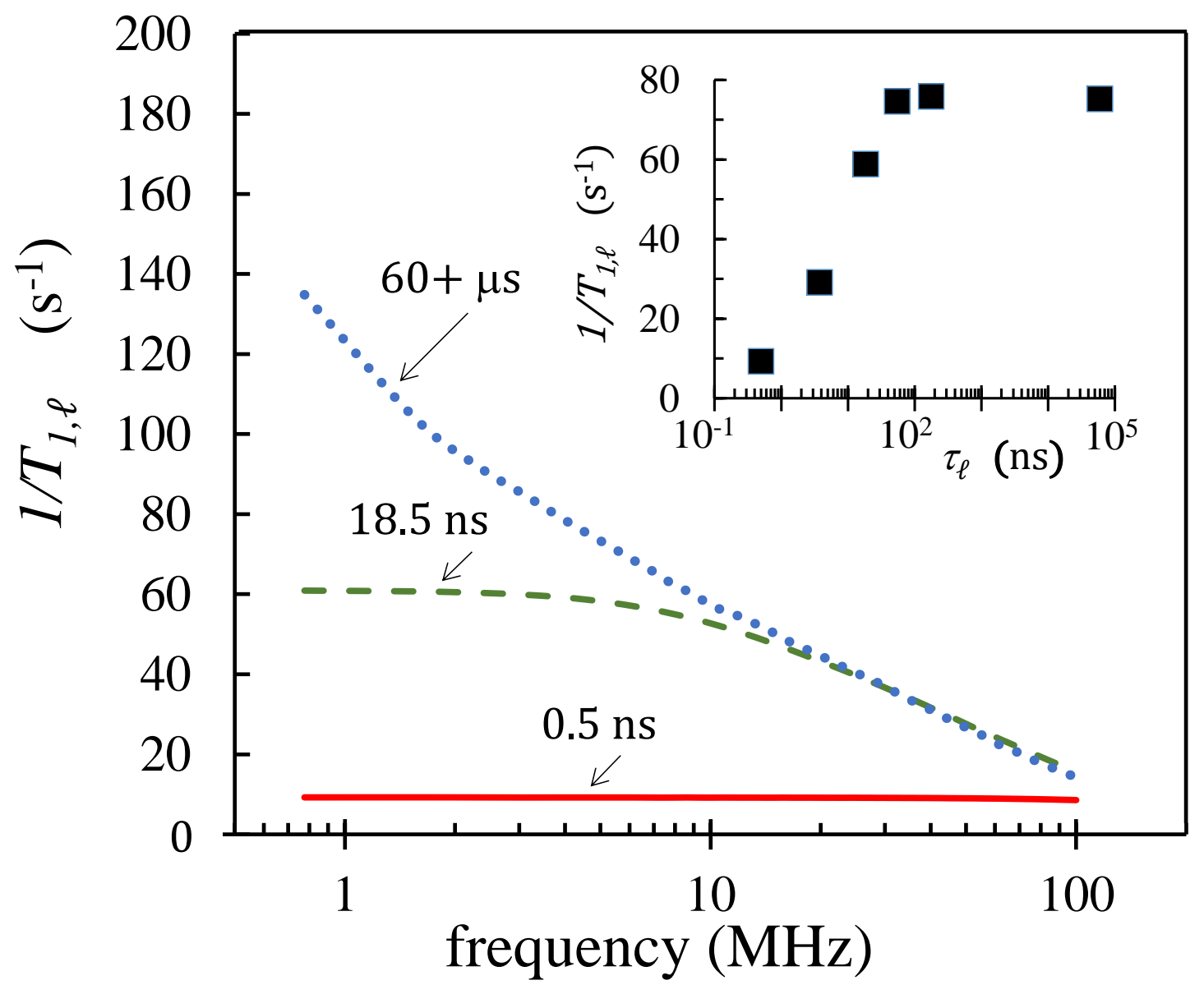

FIG. 12: The MC results for the relaxation rate contribution due to spins at the Q2D pore surfaces, $T_{1, \ell}^{-1}$, is presented for a pore of thickness $1.89 \mathrm{~nm}$ with surface desorption times $\tau_{d}=0.5 \mathrm{~ns}, 18.5 \mathrm{~ns}$ and $60+\mu \mathrm{s}$. The inset shows $T_{1, \ell}^{-1}$ as a function of the surface water diffusion correlation time $\tau_{\ell}$ at a fixed frequency of $5 \mathrm{MHz}$.

by distances larger than $r=d / 2$ at both $t=0$ and $t$, where $d$ is the simulation cell size in the $x y$ plane, and this contribution makes up an increasing fraction of $G_{\ell \ell}(t)$ as $t$ increases. Thus, we can assert that $n<0.9$ and that the Q2D pore system is an example of bulkmediated surface diffusion (BMSD) $[5,12]$. We note, however, that the MC system is set up to facilitate the observation of Lévy dynamics, that is the rate contants provide for $4 \%$ of the surface sites to be vacant and the pore thickness is small leading to frequent encounters of spins with the surface. Despite this, the Lévy effect on $G_{b b}(t)$ is weak, influences the longtime region of the curve which does not contribute significantly to $T_{1}^{-1}$ when the Fourier transform is taken, and so does not make a significant impact on the relaxation rate. 
Finally, Fig. 12 presents the contribution to the spin-lattice relaxation rate due to surface spins, $T_{1, \ell}$, for $\tau_{d}=0.5 \mathrm{~ns}, 18.5 \mathrm{~ns}$ and $60+\mu \mathrm{s}$ as a function of $f$ for the range $1-100 \mathrm{MHz}$. The relaxation rates are large due to the long diffusion correlation and the enhanced probability of repeated encounters of spins in 2D compared to 3D bulk diffusion. The inset figure shows $T_{1, \ell}$ as a function of $\tau_{\ell}$ at a frequency of $5 \mathrm{MHz} . T_{1, \ell}$ is a strong function of $\tau_{d}$ over the range $0.5-50 \mathrm{~ns}$. Thereafter, $T_{1, \ell}$ is constant at about $80 \mathrm{~s}^{-1}$.

\section{CONCLUSIONS}

A theoretical model is presented for the determination of the homonuclear dipolar correlation function $G(t)$ for the translational motion of ${ }^{1} \mathrm{H}$ spins diffusing in a Q2D pore. This is a necessary first step for the computation of the NMR relaxation rates $T_{1}^{-1}$ and $T_{2}^{-1}$. An adjustable parameter allows the model to produce the correct $T_{1}^{-1}$ for bulk water at room temperature thereby compensating for omitted relaxation effects associated with rotation and local structure. $G(t)$ is computed for pore thicknesses ranging from $1 \mathrm{~nm}$ to $50 \mathrm{~nm}$ with a diffusion correlation time of $\tau_{b}=5.3 \mathrm{ps}$ corresponding to pure water at room temperature. The theoretical calculations confirm that $G(t)$ possesses the unusual form first identified in earlier work [39], that is $G(t)$ decays as $t^{-3 / 2}$ characteristic of a 3D bulk fluid before undergoing a transition towards a $t^{-1}$ dependence at long times. The transition is associated with the dominant contribution to $G(t)$ (and hence relaxation rates) of spin pair vectors which lie in a plane parallel to the pore surfaces at both $t=0$ and at long times. The transition time is found to be approximately equal to the mean time for a spin to cross the pore.

Typical pore sizes in fluidised porous systems straddle length scales from nanometres to microns. The spin-lattice relaxation rate $T_{1}^{-1}$ is computed from $G(t)$ and found to be independent of frequency over the range $0.01-100 \mathrm{MHz}$ provided the pore thickness $h>5 \mathrm{~nm}$. $T_{1}^{-1}$ for pores of thickness $h<5 \mathrm{~nm}$ increases with decreasing frequency and decreasing $h$, reaching $0.4 \mathrm{~s}^{-1}$ for the $1 \mathrm{~nm}$ pore at $f=10 \mathrm{kHz} . T_{1}^{-1}$ is then calculated as a function of the bulk water diffusion correlation time $\tau_{b}$ for a pore of fixed thickness $5 \mathrm{~nm}$. The objective is to estimate the contribution to the observed relaxation rate due to bulk water in a porous medium in which the water exhibits a reduced effective diffusion coefficient due to the presence of solutes. We find that $T_{1}^{-1}$ increases linearly with increasing $\tau_{b}$. This result 
allows an estimate of the diffusion coefficient of the bulk fluid from the $T_{1}^{-1}$ dispersion curve for systems where the influence of paramagnetic impurities is negligible without the need for additional experimentation.

Monte Carlo simulations of hydrated Q2D pores comprising surface spins and bulk spin environments were undertaken for a pore of thickness $1.89 \mathrm{~nm}$. The MC approach enables the study of surface-bulk exchange processes not presently accessible to theoretical analysis. The surface water is assigned an in-plane diffusion coefficient of $10^{-11} \mathrm{~m}^{2} / \mathrm{s}$ and desorbs from surface sites at a characteristic rate $\tau_{d}^{-1}$. Both the bulk water and surface water contributions to the dipolar correlation function are determined.

The MC simulations show that the contribution to the relaxation rate associated with bulk water, $T_{1, b}^{-1}$, is $0.13 \pm 0.02 \mathrm{~s}^{-1}$ for a pore of thickness $1.89 \mathrm{~nm}$ and is virtually independent of both $\tau_{d}$ and frequency $f$, provided $f<100 \mathrm{MHz}$. By contrast, the dipolar correlation function (and hence the relaxation rate) associated with the surface spins, $G_{\ell \ell}(t)$, is a strong function of $\tau_{d}$. At $\tau_{d}=60+\mu s$, in which there is no exchange of spins between the surface and bulk environments, $G_{\ell \ell}(t) \propto t^{-1}$ for $t \gtrsim 1 \mathrm{~ns}$ consistent with $2 \mathrm{D}$ diffusive motion. For $\tau_{d}=0.5 \mathrm{~ns}$, the correlation function starts to decay at $t \approx \tau_{d}$ due to the loss of spins from the surface before 2D surface diffusion becomes significant. Then $G(t)$ displays an abrupt change in gradient at $t \gtrsim 5 \mathrm{~ns}$ which is associated with surface spins which desorb into the bulk and then return to the surface at a later time. This is an example of bulk-mediated surface diffusion resulting in Lévy dynamics.

Finally, the spin-lattice relaxation rate due to surface spins, $T_{1, \ell}^{-1}$, is determined from the MC simulation as a function of desorption time $\tau_{d}$ for the range $f=1-100 \mathrm{MHz}$. The relaxation rates are found to be large, up to $140 \mathrm{~s}^{-1}$ at $1 \mathrm{MHz}$, due to the long diffusion correlation time and the enhanced probability of repeated encounters of spins in $2 \mathrm{D}$ compared to $3 \mathrm{D}$ bulk diffusion. $T_{1, \ell}^{-1}$ is found to be a strong function of $\tau_{d}$ provided $\tau_{d}<50$ ns. Thereafter, $T_{1, \ell}^{-1}$ is constant at about $80 \mathrm{~s}^{-1}$.

The theoretical results allow a quantitative estimate of the NMR relaxation rates for fluidised porous systems where measured rates are not dominated by the presence of paramagnetic impurities. The theoretical calculations are validated by MC simulations which also allow the modelling of the dynamical interchange of spins between surface and bulk environ- 
ments. These provide insight as to the important contributions to measured rates for water confined to nanometre-sized pores. The theoretical results are used in a detailed analysis in Secs. IIIA and IIIB of Paper II of the experimental $T_{1}^{-1}$ dispersions for, respectively, a plaster paste [10] and a synthetic saponite clay [5]. Neither system contains paramagnetic impurities. The analysis in Paper II allows meaningful nano-scale transport properties to be obtained for the water in each system.

\section{Acknowledgments}

$\mathrm{NCH}$ acknowledges the support of Engineering and Physical Sciences Research Council (UK) for financial support (Grant number EP/H033343/1). We would also like to thank Jean-Pierre Korb for helpful discussions relating to this work.

\section{Appendix A: The determination of $P\left(z, t \cap z_{0}\right)$ for a Q2D pore}

The probability density function $P\left(z, t \cap z_{0}\right)=P\left(z_{0}\right) P\left(z, t \mid z_{0}\right)$ describes the probability per unit length of finding a pair of spins separated by $z$ at time $t$ and by $z_{0}$ at $t=0 . P\left(z, t \mid z_{0}\right)$ is the probability density function for a spin pair separated by $z$ at time $t$ given the pair was separated by $z_{0}$ at $t=0$ and $P\left(z_{0}\right)$ is the a priori probability density function per unit length describing the probability of finding a spin pair separated by $z_{0}$. A schematic diagram of the system, including a definition of the coordinates, is presented in Fig. 13.

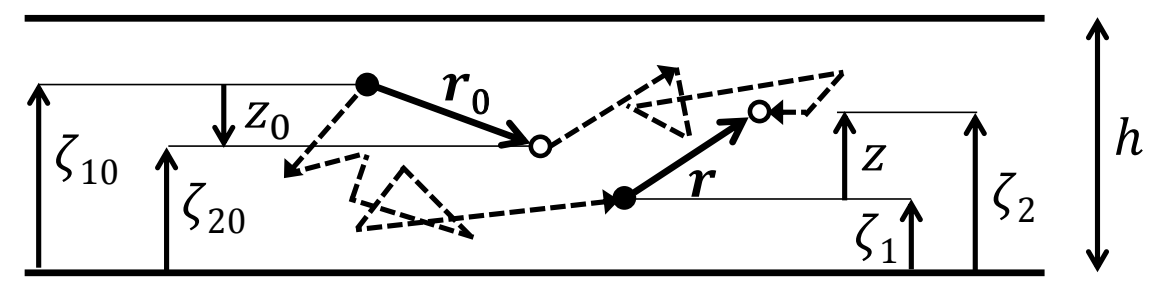

FIG. 13: The spin-pair vector $\mathbf{r}_{0}$ connecting spin $1(\bullet)$ and spin $2(0)$ at $t=0$ is indicated by $\mathbf{r}_{0}$. Spin 1 and spin 2 have coordinates in the direction perpendicular to the pore surface labelled $\zeta_{1,0}$ and $\zeta_{2,0}$ respectively. The two spins diffuse and at a time $t$ are separated by the vector $\mathbf{r}$. The spin-pair coordinate $z$ is the component of $\mathbf{r}$ in the direction perpendicular to the plane. 
$P\left(z_{0}\right)$ is obtained by evaluating the convolution $P\left(\zeta_{1,0}\right) * P\left(\zeta_{2,0}\right)$ with $z_{0}=\zeta_{2,0}-\zeta_{1,0}$ where spins 1 and 2 have coordinates $\zeta_{1,0}$ and $\zeta_{2,0}$ respectively at $t=0$ and assuming spins are uniformly distributed across the pore. The probability density functions $P\left(\zeta_{1,0}\right)$ and $P\left(\zeta_{2,0}\right)$ are uniform with a magnitude $h^{-1}$ over the range $0 \leq \zeta_{1,0}, \zeta_{2,0} \leq h$. The convolution yields

$$
P\left(z_{0}\right)=N_{\mathrm{V}}^{1 / 3}\left(1-\frac{\left|z_{0}\right|}{h}\right)
$$

where the number of spins per unit length is expressed as $N_{\mathrm{V}}^{1 / 3}$ where $N_{\mathrm{v}}$ is the spin volume density.

The probability density function $P\left(\zeta, t \mid \zeta_{0}\right)$ describing the probability that a single spin is located at the coordinate $\zeta$ at time $t$ given it was located at $\zeta_{0}$ at $t=0$ is found by a standard solution to the diffusion equation with reflective boundaries at $\zeta=0$ and $\zeta=h$ as (see, for example, Ref. [47])

$$
P\left(\zeta, t \mid \zeta_{0}\right)=\frac{1}{h}\left[1+2 \sum_{p=1}^{\infty} e^{-D p^{2} \pi^{2} t / h^{2}} \cos \frac{p \pi \zeta}{h} \cos \frac{p \pi \zeta_{0}}{h}\right]
$$

where $D$ is defined by Eq. (1). As $t \rightarrow 0, P\left(\zeta, t \mid \zeta_{0}\right)$ is a delta function located at $\zeta_{0}$ and at finite time $t$ yields a Gaussian distribution centred on $\zeta_{0}$ which spreads as $t$ increases with the probability density reflecting at the boundaries $\zeta=0$ and $\zeta=h$. The probability function $P\left(z, t \mid z_{0}\right)$ is found by taking the double convolution

$$
P\left(z, t \mid z_{0}\right)=\iint P\left(\zeta_{2}, t \mid \zeta_{2,0}\right) P\left(\zeta_{2}-z, t \mid \zeta_{2,0}-z_{0}\right) d \zeta_{2} d \zeta_{2,0}
$$

where $z=\zeta_{2}-\zeta_{1}$ and $z_{0}=\zeta_{2,0}-\zeta_{1,0}$. Eq. (A2) is then substituted into Eq. (A3) but care is required in executing the integrations which are best performed separately for regions $z<0$ and $z>0$ (also for regions $z_{0}<0$ and $z_{0}>0$ ) where the integration limits are $\zeta_{2}=0 \ldots z+h$ and $\zeta_{2}=z \ldots h$ respectively. Moreover, for a given $z_{0}$, the coordinate $\zeta_{1,0}$ is limited to the range $0 \ldots h-z_{0}$ if $z_{0}$ is positive and to $-z_{0} \ldots h$ if $z_{0}$ is negative. In each case the probability density function for $\zeta_{1,0}$ is uniform with a magnitude $\left(h-\left|z_{0}\right|\right)^{-1}$. Expressing the final result 
in terms of dimensionless quantities $w=z / \delta$ and $\Delta=h / \delta$ yields, eventually,

$$
\begin{aligned}
P\left(w, t \mid w_{0}\right)= & \frac{1}{\Delta-\left|w_{0}\right|}\left[d(w) d\left(w_{0}\right)+\frac{4}{\pi^{2}} \sum_{p=1}^{\infty} \frac{E_{p}(t)}{p^{2}} \operatorname{sgn}\left(w w_{0}\right)^{p} s_{p}(w) s_{p}\left(w_{0}\right)\right. \\
& +\sum_{p=1}^{\infty} E_{p}(2 t) f_{p}(w) f_{p}\left(w_{0}\right)+\frac{4}{\pi^{2}} \sum_{p=1}^{\infty} \sum_{q>p}^{\infty} \frac{E_{p}(t) E_{q}(t) \Phi_{p q}\left(w w_{0}\right)}{\left(p^{2}-q^{2}\right)^{2}} \\
& \left.\times\left[p s_{p}(w)+\chi_{p q} q s_{q}(w)\right]\left[p s_{p}\left(w_{0}\right)+\chi_{p q} q s_{q}\left(w_{0}\right)\right]\right]
\end{aligned}
$$

where

$$
\begin{array}{cc}
E_{p}(t)=e^{-p^{2} \pi^{2} t / 6 \Delta^{2} \tau} & d(w)=1-\frac{|w|}{\Delta} \quad s_{p}(w)=\sin \frac{p \pi|w|}{\Delta} \\
c_{p}(w)=\cos \frac{p \pi|w|}{\Delta} & f_{p}(w)=d(w) c_{p}(w)-\frac{s_{p}(w)}{p \pi}
\end{array}
$$

where $\Phi_{p q}\left(w w_{0}\right)=2$ if $w$ and $w_{0}$ have the same sign, $\Phi_{p q}\left(w w_{0}\right)=-2 \chi_{p q}$ if $w$ and $w_{0}$ have opposite signs. Here $\chi_{p q}=1$ if $p+q$ is an odd number and $\chi_{p q}=-1$ if $p+q$ is even. Rewriting Eq. (A1) in terms of $w_{0}$ and $\Delta$ and multiplying by Eq. (A4) produces the final expression for $P\left(w, t \cap w_{0}\right)$,

$$
\begin{aligned}
P\left(w, t \cap w_{0}\right)= & \frac{N_{\mathrm{v}}^{1 / 3}}{\delta \Delta}\left[d(w) d\left(w_{0}\right)+\frac{4}{\pi^{2}} \sum_{p=1}^{\infty} \frac{E_{p}(t)}{p^{2}} \operatorname{sgn}\left(w w_{0}\right)^{p} s_{p}(w) s_{p}\left(w_{0}\right)\right. \\
& +\sum_{p=1}^{\infty} E_{p}(2 t) f_{p}(w) f_{p}\left(w_{0}\right)+\frac{4}{\pi^{2}} \sum_{p=1}^{\infty} \sum_{q>p}^{\infty} \frac{E_{p}(t) E_{q}(t) \Phi_{p q}\left(w w_{0}\right)}{\left(p^{2}-q^{2}\right)^{2}} \\
& \left.\times\left\{\left[p s_{p}(w)+\chi_{p q} q s_{q}(w)\right]\left[p s_{p}\left(w_{0}\right)+\chi_{p q} q s_{q}\left(w_{0}\right)\right]\right\}\right]
\end{aligned}
$$

\section{Appendix B: The evaluation of the spin-pair integrals}

In the expression for $G(t)$ given by Eq. (11), the evaluation of four integrals with respect to variables $u, w, u_{0}$ and $w_{0}$ is required for each of $M=0,1$ and 2 , where $u=\rho / \delta$ and $w=z / \delta$ are dimensionless variables. This appendix executes these integrals where analytic and provides expressions to be evaluated numerically in the cases where an analytic expression is not possible or practical. 
A schematic diagram of the spin-pair vector space is presented in Fig. 2 in which the spin-pair vectors are identified by cylindrical coordinates. The spatial integrals of Eq. (11) may be written

$$
\int_{\mathbb{R}^{2}} \int_{\mathbb{R}_{0}^{2}}\left[\sum_{M=0}^{2} h_{M}\left(\kappa, u_{0}, w_{0}\right) h_{M}(\kappa, u, w)\right] P\left(w, t \cap w_{0}\right) d u_{0} d w_{0} d u d w
$$

where $h_{M}(\kappa, u, w)$ is given by Eq. (12). The pore volume is divided into two regions labelled $\mathrm{A}$ and $\mathrm{B}$ in Fig. 2. Region $\mathrm{A}$, in dimensionless units, represents the space $-\Delta \leq w \leq-\Delta^{\prime}$ and $\Delta^{\prime} \leq w \leq \Delta$ with $0 \leq u \leq \infty$ where $\Delta=h / \delta$ is the pore thickness in units of $\delta$ and $\Delta^{\prime}=\delta^{\prime} / \delta$. Region $\mathrm{B}$ is defined by $-\Delta^{\prime} \leq w \leq \Delta^{\prime}$ and $\Delta^{\prime} \leq u \leq \infty$. The cylindrical volume of radius and half-height $\Delta^{\prime}$ is therefore excluded from the integrations.

The integrals given by Eq. (B1) become

$$
\int_{\mathbb{R}^{2}} \int_{\mathbb{R}_{0}^{2}} \cdots=\frac{N_{\mathrm{V}}^{1 / 3}}{\delta \Delta}\left[I_{\mathrm{AA}}(\kappa, t)+I_{\mathrm{AB}}(\kappa, t)+I_{\mathrm{BA}}(\kappa, t)+I_{\mathrm{BB}}(\kappa, t)\right] .
$$

where the two subscripts label the integration region at time $t=0$ and at time $t$ respectively. Thus $I_{\mathrm{AA}}(\kappa, t)$, accounts for spin pairs for which $\Delta^{\prime} \leq|w| \leq \Delta$ both at $t=0$ and at time $t$. Thus, $I_{\mathrm{XY}}(\kappa, t)$ (where $\mathrm{X}$ and $\mathrm{Y}$ each refer to either region $\mathrm{A}$ or $\mathrm{B}$ ) may be written

$$
\begin{aligned}
I_{\mathrm{XY}}(\kappa, t)= & 4 D_{M, \mathrm{X}}(\kappa) D_{M, \mathrm{Y}}(\kappa)+\frac{8}{\pi^{2}} \sum_{p=1}^{\infty} \frac{E_{p}(t)}{p^{2}} \epsilon_{M}(p) S_{M, \mathrm{X}}(p, \kappa) S_{M, \mathrm{Y}}(p, \kappa) \\
& +4 \sum_{p=1}^{\infty} E_{p}(2 t) F_{M, \mathrm{X}}(p, \kappa) F_{M, \mathrm{Y}}(p, \kappa)+\frac{32}{\pi^{2}} \sum_{p=1}^{\infty} \sum_{q>p}^{\infty} \frac{E_{p}(t) E_{q}(t)}{\left(p^{2}-q^{2}\right)^{2}} \epsilon_{M}(p+q) \\
& \times\left\{\left[p S_{M, \mathrm{X}}(p, \kappa)+\chi_{p q} q S_{M, \mathrm{X}}(q, \kappa)\right]\left[p S_{M, \mathrm{Y}}(p, \kappa)+\chi_{p q} q S_{M, \mathrm{Y}}(q, \kappa)\right]\right\}
\end{aligned}
$$

where the repeated subscript $M$ implies summation for $M=0,1$, and 2 , and where $\epsilon_{M}(p)$ has the property that $\epsilon_{2}(p)=\epsilon_{0}(p)=1$ if $p$ is even and 0 if $p$ is odd, $\epsilon_{1}(p)=1$ if $p$ is odd and 0 if $p$ is even. Equation (B3) has been expressed so that the $D, F$ and $S$ functions are

$$
\begin{aligned}
D_{M, \mathrm{X}}(\kappa) & =\int_{\mathrm{X}+} h_{M}(\kappa, u, w) d(w) d u d w \\
F_{M, \mathrm{X}}(p, \kappa) & =\int_{\mathrm{X}+} h_{M}(\kappa, u, w) f_{p}(w) d u d w \\
S_{M, \mathrm{X}}(p, \kappa) & =\int_{\mathrm{X}+} h_{M}(\kappa, u, w) s_{p}(w) d u d w
\end{aligned}
$$

where $X+$ indicates that the integration with respect to $w$ is for the case where $w>0$ only. 
The $d, f$, and $s$ functions are presented as Eq. (A5).

The $D, F$, and $S$ functions are required for each region $\mathrm{A}$ and $\mathrm{B}$ for $M=0,1$, and 2 . For region $\mathrm{A}$, the integrations with respect to $u$ and $w$ are analytic and yield

$$
\begin{aligned}
& D_{2, \mathrm{~A}}(\kappa)=\frac{\sqrt{5 \pi}}{\sqrt{12} \kappa \Delta}\left[\kappa e^{-\kappa \Delta^{\prime}}\left(\Delta-\Delta^{\prime}\right)+e^{-\kappa \Delta}-e^{-\kappa \Delta^{\prime}}\right] \\
& D_{1, \mathrm{~A}}(\kappa)=0 \quad D_{0, \mathrm{~A}}(\kappa)=\sqrt{3} D_{2, \mathrm{~A}}(\kappa)
\end{aligned}
$$

and

$$
\begin{array}{r}
F_{2, \mathrm{~A}}(p, \kappa)=\frac{\sqrt{5 \pi} \kappa}{\sqrt{3} a_{p+}^{2}(\kappa)}\left[\kappa \Delta e^{-\kappa \Delta^{\prime}} c\left(p, \Delta^{\prime}\right)\left[a_{p+}(\kappa)\left(\Delta-\Delta^{\prime}\right)-2 \kappa \Delta^{2}\right]+2 \kappa^{2} \Delta^{3} e^{-\kappa \Delta}(-1)^{p}\right. \\
\left.-\frac{e^{-\kappa \Delta^{\prime}} s\left(p, \Delta^{\prime}\right)}{p \pi}\left[\kappa \Delta^{2} a_{p-}(\kappa)+p^{2} \pi^{2}\left(\Delta-\Delta^{\prime}\right) a_{p+}(\kappa)\right]\right] \\
F_{1, \mathrm{~A}}(p, \kappa)=0 \quad F_{0, \mathrm{~A}}(p, \kappa)=\sqrt{3} F_{2, \mathrm{~A}}(\kappa)
\end{array}
$$

where $a_{p \pm}(\kappa)=\kappa^{2} \Delta^{2} \pm p^{2} \pi^{2}$. Finally, for region $\mathrm{A}$,

$$
\begin{aligned}
& S_{2, \mathrm{~A}}(p, \kappa)=\frac{\sqrt{5 \pi} \kappa \Delta}{\sqrt{12} a_{p+}(\kappa)}\left[\kappa \Delta s\left(p, \Delta^{\prime}\right) e^{-\kappa \Delta^{\prime}}-(-1)^{p} p \pi e^{-\kappa \Delta}+p \pi c\left(p, \Delta^{\prime}\right) e^{-\kappa \Delta^{\prime}}\right] \\
& S_{1, \mathrm{~A}}(p, \kappa)=2 S_{2, \mathrm{~A}}(p, \kappa) \quad S_{0, \mathrm{~A}}(p, \kappa)=\sqrt{3} S_{2, \mathrm{~A}}(\kappa)
\end{aligned}
$$

Numerical solutions to the $D, F$, and $S$ functions for region B are required. A satisfactory approach is to, first, complete the integrations with respect to $u$. For the special case $w=0$, the integrals

$$
H_{M}(\kappa, 0)=\int_{\Delta^{\prime}}^{\infty} h_{M}(\kappa, u, 0) d u
$$

may be expressed

$$
\begin{aligned}
& H_{2}(\kappa, 0)=\frac{1}{2} \sqrt{15 \pi} \kappa \int_{\kappa \Delta^{\prime}}^{\infty} \frac{J_{2}(v)}{v^{2}} d v \\
& H_{1}(\kappa, 0)=0 \\
& H_{0}(\kappa, 0)=-\frac{1}{2} \sqrt{5 \pi} \kappa \int_{\kappa \Delta^{\prime}}^{\infty} \frac{J_{0}(v)}{v^{2}} d v
\end{aligned}
$$


where an upper limit on the numerical integrals of $v \approx 20$ suffices. For $w>0$

$$
\begin{aligned}
& H_{2}(\kappa, w)=\frac{1}{2} \sqrt{15 \pi}\left[\frac{\kappa e^{-\kappa|w|}}{3}-\int_{0}^{\Delta^{\prime}} \frac{J_{2}(\kappa u) u^{3}}{\left(u^{2}+w^{2}\right)^{5 / 2}} d u\right] \\
& H_{1}(\kappa, w)=\sqrt{15 \pi} \operatorname{sgn}(w)\left[\frac{\kappa e^{-\kappa|w|}}{3}-\int_{0}^{\Delta^{\prime}} \frac{J_{1}(\kappa u) w u^{2}}{\left(u^{2}+w^{2}\right)^{5 / 2}} d u\right] \\
& H_{0}(\kappa, w)=\frac{1}{2} \sqrt{5 \pi}\left[\kappa e^{-\kappa|w|}-\int_{0}^{\Delta^{\prime}} \frac{J_{0}(\kappa u) u\left(2 w^{2}-u^{2}\right)}{\left(u^{2}+w^{2}\right)^{5 / 2}} d u\right] .
\end{aligned}
$$

The numerical integrations are straightforward due to the short integration distance $0 . . \Delta^{\prime}$. Indeed, as the $H$ functions are well-behaved, it is sufficient to determine $H_{M}(\kappa, w)$ for $w=$ $0, \frac{1}{4} \Delta^{\prime}, \frac{1}{2} \Delta^{\prime}, \frac{3}{4} \Delta^{\prime}$ and $\Delta^{\prime}$ with interpolation between points if necessary. Thus,

$$
\begin{aligned}
D_{M, \mathrm{~B}}(\kappa) & =\int_{0}^{\Delta^{\prime}} H_{M}(\kappa, w) d(w) d w \\
F_{M, \mathrm{~B}}(p, \kappa) & =\int_{0}^{\Delta^{\prime}} H_{M}(\kappa, w) f_{p}(w) d w \\
S_{M, \mathrm{~B}}(p, \kappa) & =\int_{0}^{\Delta^{\prime}} H_{M}(\kappa, w) s_{p}(w) d w
\end{aligned}
$$

noting that $D_{1, \mathrm{~B}}(\kappa)=F_{1, \mathrm{~B}}(p, \kappa)=0$. 
[1] S. Stapf, R. Kimmich, and R.-O. Seitter, Phys. Rev. Lett. 75, 2855 (1995).

[2] R. Valiullin, R. Kimmich, and N. Fatkullin, Phys. Rev. E 56, 4371 (1997).

[3] J.-P. Korb, M. Whaley-Hodges, and R. G. Bryant, Phys. Rev. E 56, 1934 (1997).

[4] J.-P. Korb, M. Whaley-Hodges, T. Gobron, and R. G. Bryant, Phys. Rev. E 60, 3097 (1999).

[5] T. Zavada, R. Kimmich, G. J., and K. A., J. Chem. Phys. 110, 6977 (1999).

[6] S. Godefroy, J.-P. Korb, M. Fleury, and R. G. Bryant, Phys. Rev. E 64, 021605 (2001).

[7] F. Barberon, J.-P. Korb, D. Petit, V. Morin, and E. Bermejo, Phys. Rev. Lett. 90, 116103 (2003).

[8] L. Monteilhet, J.-P. Korb, J. Mitchell, and P. J. McDonald, Phys. Rev. E 74, 061404 (2006).

[9] J.-P. Korb, P. J. McDonald, L. Monteilhet, A. G. Kalinichev, and R. J. Kirkpatrick, Cement Concrete Res. 37, 348 (2007).

[10] J.-P. Korb, New J. Phys. 13, 035016 (2011).

[11] J.-P. Korb, B. Nicot, A. Louis-Joseph, S. Bubici, and G. Ferrante, J. Phys. Chem. C 118, $23212(2014)$.

[12] R. Kimmich, Chem. Phys. 284, 253 (2002).

[13] A. Plassais, M.-P. Pomiés, N. Lequeux, J.-P. Korb, D. Petit, F. Barberon, and B. Bresson, Phys. Rev. E 72, 041401 (2005).

[14] A. E. Sitnitsky, G. G. Pimenov, and A. V. Anisimov, J. Magn. Reson. 172, 48 (2005).

[15] P. J. McDonald, J.-P. Korb, J. Mitchell, and L. Monteilhet, Phys. Rev. E 72, 011409 (2005).

[16] L. F. Gladden and J. Mitchell, New J. Phys. 13, 035001 (2011).

[17] V.-V. Telkki, M. Yliniemi, and J. Jokisaari, Holzforschung 67, 291 (2013).

[18] .

[19] D. N. Winslow and S. Diamond, Journal of The American Ceramic Society 57, 193 (1974).

[20] D. N. Winslow, J. M. Bukowski, and J. Francis Young, Cement and concrete research 24, 1025 (1994).

[21] S. Stock, N. Naik, A. Wilkinson, and K. Kurtis, Cement and Concrete Research 32, 1673 (2002).

[22] K. L. Scrivener, T. Füllmann, E. Gallucci, G. Walenta, and E. Bermejo, Cement and Concrete 
Research 34, 1541 (2004).

[23] J. J. Thomas, H. M. Jennings, and A. J. Allen, Cement and Concrete Research 28, 897 (1998).

[24] E. Fratini, S.-H. Chen, P. Baglioni, and M.-C. Bellissent-Funel, J. Phys. Chem. B 106, 158 (2002).

[25] A. J. Allen, J. J. Thomas, and H. M. Jennings, Nat. Mater. 6, 311 (2007).

[26] A. J. Allen and J. J. Thomas, Cement and Concrete Research 37, 319 (2007).

[27] H. N. Bordallo, L. P. Aldridge, and A. Desmedt, The Journal of Physical Chemistry B 110, 17966 (2006).

[28] N. Bloembergen, E. M. Purcell, and R. V. Pound, Phys. Rev. 73, 679 (1948).

[29] P. Levitz, M. Zinsmeister, P. Davidson, D. Constantin, and O. Poncelet, Phys. Rev. E 78, $030102(2008)$.

[30] O. Bénichou, D. Grebenkov, P. Levitz, C. Loverdo, and R. Voituriez, Phys. Rev. Lett. 105, $150606(2010)$.

[31] O. Bénichou, D. Grebenkov, P. Levitz, C. Loverdo, and R. Voituriez, J. Stat. Phys. 142, 657 (2011).

[32] O. V. Bychuk and B. O’Shaughnessy, Langmuir 10, 3260 (1994).

[33] O. V. Bychuk and B. O’Shaughnessy, Phys. Rev. Lett. 74, 1795 (1995).

[34] J.-P. Korb, S. Xu, and J. Jonas, J. Chem. Phys. 98, 2411 (1993).

[35] M. Holz, S. R. Heil, and A. Sacco, Physical Chemistry Chemical Physics 2, 4740 (2000).

[36] P. Tofts, D. Lloyd, C. Clark, G. Barker, G. Parker, P. McConville, C. Baldock, and J. Pope, Magnetic resonance in medicine 43, 368 (2000).

[37] D. A. Faux and P. J. McDonald, Phys. Rev. Fluids (2016).

[38] A. Abragam, The Principles of Nuclear Magnetism (Oxford University Press, 1961).

[39] D. A. Faux, P. J. McDonald, N. C. Howlett, J. S. Bhatt, and S. V. Churakov, Phys. Rev. E 87, 062309 (2013).

[40] C. Calero, J. Martí, and E. Guàrdia, J. Phys. Chem. B 119, 1966 (2015).

[41] A. LeClaire and A. Lidiard, Philosophical magazine 1, 518 (1956).

[42] R. Tahir-Kheli and R. Elliott, Physical Review B 27, 844 (1983).

[43] K. Krynicki, C. D. Green, and D. W. Salter, Faraday Discuss. Chem. Soc. 66, 199 (1978).

[44] D. A. Faux, P. J. McDonald, N. C. Howlett, J. S. Bhatt, and S. V. Churakov, Phys. Rev. E 
91, $032311(2015)$.

[45] D. A. Faux, D. K. Ross, and C. A. Sholl, J. Phys. C: Solid State Phys. 19, 4115 (1986).

[46] D. S. Grebenkov, Y. A. Goddard, G. Diakova, J.-P. Korb, and R. G. Bryant, The Journal of Physical Chemistry B 113, 13347 (2009).

[47] T. Bickel, Physica A 377, 24 (2007).

[48] The cut-off could be less than $d / 2$ but not more due to correlation effects associated with periodic boundaries

[49] In real systems, as explored in Paper II, exchange averaging applies. In systems with intermediate exchange rate, this is not immediately apparent and can lead to erroneous conclusions. Further, in porous media, background magnetic field gradients can lead to signal attenuation dependent on the experimental parameters (most notably the CPMG pulse gap in $T_{2}$ measurements). Finally, CPMG experiments with very short pulse gap, spin-locking $\left(T_{1 \rho}\right)$ may be manifest. None of these largely experimental factors are considered here save to note that a combination of relaxation and diffusion experiments can often resolve ambiguities. In particular, measurements of diffusivity can provide an independent check on translational correlation times. 